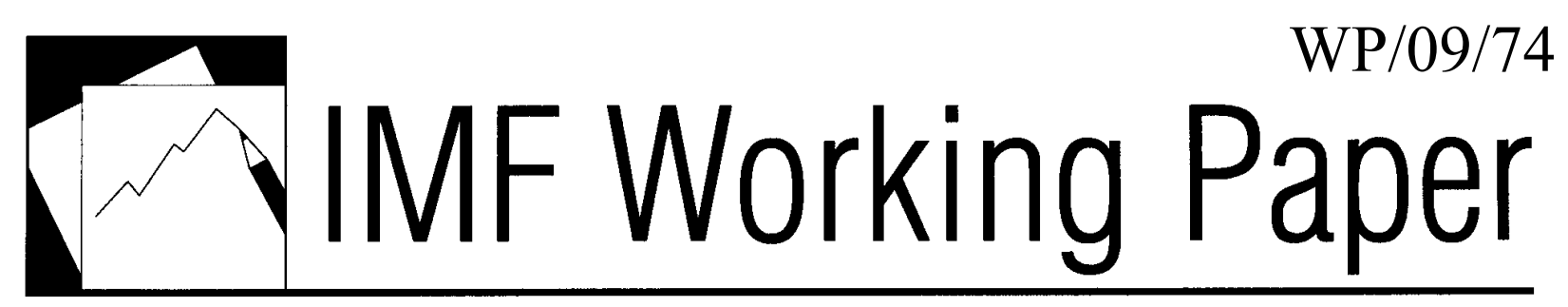

\title{
Limited Information Bayesian Model Averaging for Dynamic Panels with Short Time Periods
}

Huigang Chen, Alin Mirestean, and Charalambos Tsangarides 



\title{
IMF Working Paper
}

Research Department

\section{Limited Information Bayesian Model Averaging for Dynamic Panels with Short Time Periods}

\section{Prepared by Huigang Chen, Alin Mirestean, and Charalambos Tsangarides ${ }^{1}$}

Authorized for distribution by Atish Ghosh

April 2009

\begin{abstract}
This Working Paper should not be reported as representing the views of the IMF. The views expressed in this Working Paper are those of the author and do not necessarily represent those of the IMF or IMF policy. Working Papers describe research in progress by the authors and are published to elicit comments and to further debate.
\end{abstract}

Bayesian Model Averaging (BMA) provides a coherent mechanism to address the problem of model uncertainty. In this paper we extend the BMA framework to panel data models where the lagged dependent variable as well as endogenous variables appear as regressors. We propose a Limited Information Bayesian Model Averaging (LIBMA) methodology and then test it using simulated data. Simulation results suggest that asymptotically our methodology performs well both in Bayesian model selection and averaging. In particular, LIBMA recovers the data generating process very well, with high posterior inclusion probabilities for all the relevant regressors, and parameter estimates very close to the true values. These findings suggest that our methodology is well suited for inference in dynamic panel data models with short time periods in the presence of endogenous regressors under model uncertainty.

JEL Classification Numbers: C11, C15, C23, C52.

Keywords: Bayesian Model Averaging; Model Uncertainty; Dynamic Panels; Generalized Method of Moments; Robustness.

Author's E-Mail Address: hchen@imf.org; amirestean@imf.org; ctsangarides@imf.org.

\footnotetext{
${ }^{1}$ We thank Steve Durlauf, Rex Ghosh, Eduardo Ley, Enrique Moral-Benito, Chris Papageorgiou, and Roberto Samaniego for helpful comments and suggestions.
} 


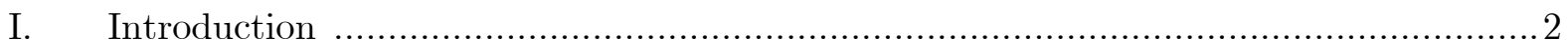

II. Model Uncertainty in the Bayesian Context .......................................................... 3

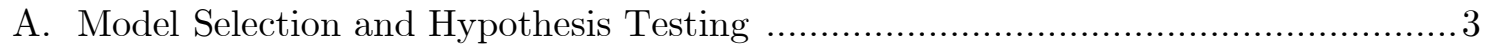

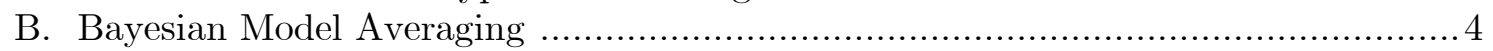

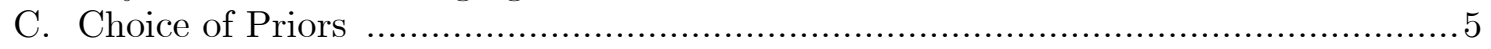

III. Limited Information Bayesian Model Averaging ................................................ 7

A. A Dynamic Panel Data Model with Endogenous Regressors ...............................

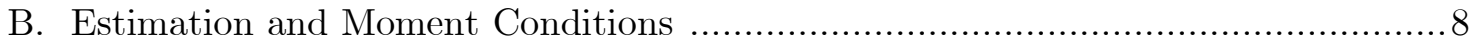

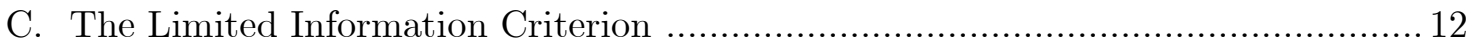

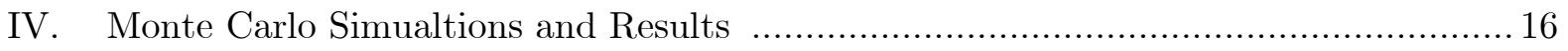

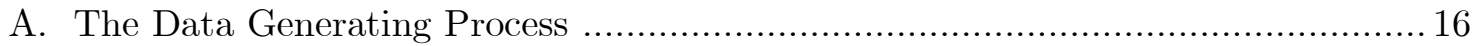

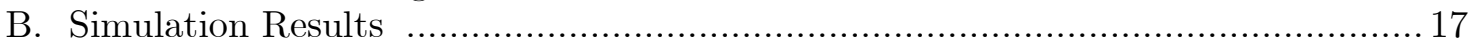

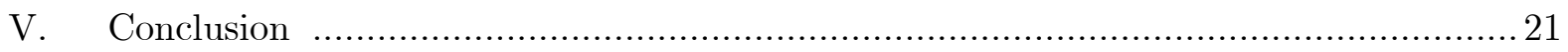

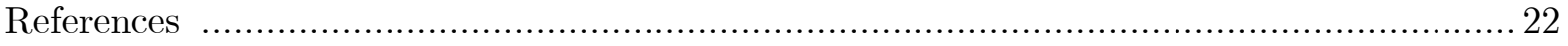

Tables:

1. Posterior Probability of the True Model ….................................................. 27

2. Posterior Probability Ratio of True Model/Best among the Other Models ..............28

3. $\quad$ Probability of Retrieving the True Model …................................................. 29

4. Model Recovery: Medians and Variances of Posterior Inclusion Probability for Each

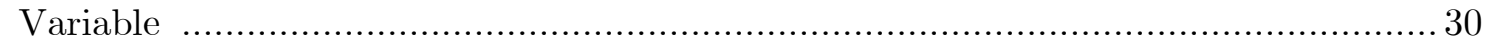

5. Model Recovery: Medians and Variances of Estimated Parameter Values ................ 31

6. Posterior Probability of the True Model (Non-Gaussian Case) …........................... 32

7. Posterior Probability Ratio: True Model/best among the Other Models (Non-Gaussian

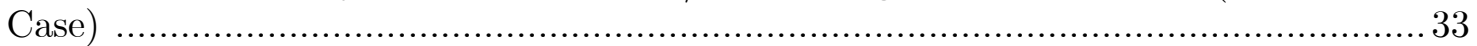

8. Probability of Retrieving the True Model (Non-Gaussian Case).............................. 34

9. Model Recovery: Medians and Variances of Posterior Inclusion Probability for Each Variable (Non-Gaussian Case) ........................................................................ 35

10. Model Recovery: Medians and Variances of Estimated Parameter Values (Non-

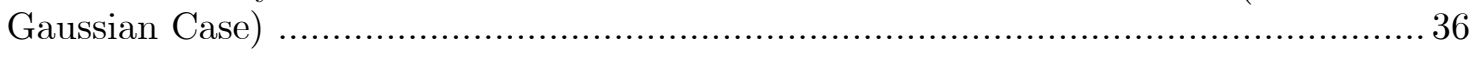

Appendix A Figures:

1. Posterior Densities for the Probabilities in Table 1 ......................................... 37

2. Posterior Densities for the Probabilities in Table 2 ......................................... 38

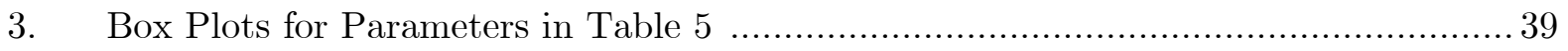

4. Posterior Densities for the Probabilities in Table 6 ........................................ 40

5. Posterior Densities for the Probabilities in Table 7 ..................................... 41

6. Box Plots for Parameters in Table 10 .............................................................. 42 


\section{INTRODUCTION}

Model uncertainty is an issue encountered often in the econometric study of socioeconomic phenomena. Initially pointed out by Leamer (1978) and later elaborated by Durlauf and Quah (1999) model uncertainty arises because the lack of clear theoretical guidance and tradeoffs on the choice of regressors result in a broad number of possible specifications, and often contradictory conclusions. In addition, attempts to deal with model uncertainty by engaging in unsystematic searches of possible model configurations, may result in overconfident and often fragile inferences. As a result, a growing number of researchers are turning to the Bayesian Model Averaging (BMA) framework in order to deal with the problem of model uncertainty.

Conceptually, BMA bases inferences on a weighted average of the full model space instead of on one selected model, and thus incorporates uncertainty in both predictions and parameter estimates. ${ }^{2}$ Seminal contributions to BMA include those of Moulton (1991), Madigan and Raftery (1994), Kass and Raftery (1995), Raftery (1995), and Raftery, Madigan and Hoeting (1997). The BMA framework has been applied in various areas of social sciences. ${ }^{3}$ In economics, some of the most notable work includes Brock and Durlauf (2001), Fernández, Ley and Steel (2001a), and Sala-i-Martin, Doppelhofer and Miller (2004). Despite the increasing interest in BMA, most of the work thus far uses static models, focusing mainly on cross section analysis with data averaged over the time dimension, thus ignoring dynamic relationships among variables. ${ }^{4}$ Moreover, to the best of our knowledge, none of the models allow for the inclusion of endogenous variables. ${ }^{5}$

In this paper we propose a methodology for dealing with model uncertainty in the context of panel data model with short time periods where the lagged dependent variable and endogenous variables appear as regressors. We use a limited information approach which refines the limited information version of Bayesian Model Averaging (LIBMA) introduced by Tsangarides (2004). The limited information criterion proposed in this paper resembles the

\footnotetext{
${ }^{2}$ In contrast to BMA, Bayesian model selection uses information criteria to select one model (one set of variables) from a set of potential models.

3 These include biology (Yeung, Bumgarner, and Raftery (2005)), ecology (Wintle et al. (2003)), public health (Morales et al. (2006)), and toxicology (Koop and Tole (2004)).

${ }^{4}$ Moral-Benito (2007) considers a panel data model where the lagged dependent variable is correlated with the individual effects but not correlated with the error term.

${ }^{5}$ By endogenous variables we understand variables that would be correlated with the contemporary error term.
} 
BIC model and moment selection criterion (MMSC-BIC) proposed by Andrews and Lu (2001), and parallels the one proposed by Hong and Preston (2008). One key difference of our approach is that we construct the likelihood by data transformation and straightforward Bayesian arguments. ${ }^{6}$ We also investigate the performance of the proposed framework relative to both Bayesian model selection and averaging by performing Monte Carlo simulations.

The remainder of the paper is organized as follows. In Section 2 we introduce the concept of model uncertainty in the Bayesian context and then review model selection and model averaging. Section 3 develops the theoretical framework of the LIBMA methodology in the context of dynamic panels with endogenous regressors. It includes the model setup, the moment conditions, the limited information criterion, and estimation. Section 4 discusses the proposed simulation experiment and presents the results. The final section concludes.

\section{MODEL UNCERTAINTY IN THE BAYESIAN CONTEXT}

For completeness, this section reviews briefly the basic theory of uncertainty in the Bayesian context. Excellent reviews include Hoeting, Madigan, Raftery and Volinsky (1999), and Chipman, George and McCulloch (2001).

\section{A. Model Selection and Hypothesis Testing}

Consider the standard linear regression model

$$
Y=\breve{Z} \theta+u
$$

where $Y$ is the variable of interest, $\breve{Z}$ is a matrix of explanatory variables, $\theta$ is a vector of unknown parameters and $u$ is the error term. Suppose there is a universe of $k$ possible explanatory variables indexed by $\mathbf{U}=\{1,2, \ldots, j, j+1, \ldots, k\}$. Let $\mathrm{Z}$ be the matrix of all possible explanatory variables. For a given model $M_{j}$ that considers only a subset of the possible explanatory variables, $M_{j} \subset \mathbf{U}$, let $\mathbf{C}_{M_{j}}=\left\{c_{m n, M_{j}}\right\}_{m, n=1}^{k}$ be a $k \times k$ diagonal

\footnotetext{
${ }^{6}$ Another related approach to derive Bayesian posteriors in models with moment conditions is by using information projection onto a family of probability measures. Kim (2002) uses a transformation of the GMM objective function to obtain a likelihood that is then used for deriving the posterior. Ragusa (2008) uses the projection of a reference distribution into the space of distributions that are consistent with the average form of the moment conditions and then uses formal Bayesian calculus to obtain the posterior.
} 
choice matrix such that its diagonal will have 1's if the corresponding variable is included in the model and 0's otherwise. Hence $c_{i i, M_{j}}=1\left\{i \in M_{j}\right\}$. Therefore, for a given model $M_{j}$, $\breve{\mathbf{Z}}=\mathbf{Z C}_{M_{j}}$ and model (1) can be now written more generally as

$$
Y=\mathbf{Z C}_{M_{j}} \theta+u
$$

where $\theta=\left(\begin{array}{llll}\theta_{1} & \theta_{2} & \ldots & \theta_{k}\end{array}\right)^{\prime}$ is the set of parameters to be estimated.

Given the universe of $k$ possible explanatory variables, a set of $K=2^{k}$ models $\mathcal{M}=\left(M_{1}, \ldots, M_{K}\right)$ are under consideration. In the spirit of Bayesian inference, one can specify priors $p\left(\theta \mid M_{j}\right)$ for the parameters of each model, and a prior $p\left(M_{j}\right)$ for each model in the model space $\mathcal{M}$.

Model selection seeks to find the model $M_{j}$ in $\mathcal{M}=\left(M_{1}, \ldots, M_{K}\right)$ that actually generated the data. Let $D=\left(\begin{array}{ll}\mathbf{Y} & \mathbf{Z}\end{array}\right)$ denote the data set available to the researcher. The probability that $M_{j}$ is the correct model, given the data $D$, is, by Bayes' rule

$$
p\left(M_{j} \mid D\right)=\frac{p\left(D \mid M_{j}\right) p\left(M_{j}\right)}{\sum_{l=1}^{K} p\left(D \mid M_{l}\right) p\left(M_{l}\right)}
$$

where

$$
p\left(D \mid M_{j}\right)=\int p\left(D \mid \theta_{j}, M_{j}\right) p\left(\theta_{j} \mid M_{j}\right) d \theta_{j}
$$

is the marginal probability of the data given model $M_{j}$.

Based on the posterior probabilities, the comparison of model $M_{j}$ against $M_{i}$ is expressed by the posterior odds ratio $\frac{p\left(M_{j} \mid D\right)}{p\left(M_{i} \mid D\right)}=\frac{p\left(D \mid M_{j}\right)}{p\left(D \mid M_{i}\right)} \cdot \frac{p\left(M_{j}\right)}{p\left(M_{i}\right)}$. Essentially, the data updates the prior odds ratio $\frac{p\left(M_{j}\right)}{p\left(M_{i}\right)}$ through the Bayes factor $\frac{p\left(D \mid M_{j}\right)}{p\left(D \mid M_{i}\right)}$ to measure the extent to which the data support $M_{j}$ over $M_{i}$. When the posterior odds ratio is greater (less) than 1 the data favor $M_{j}$ over $M_{i}\left(M_{i}\right.$ over $\left.M_{j}\right)$. Often the prior odds ratio is set to 
1 representing the lack of preference for either model, ${ }^{7}$ in which case the posterior odds ratio is equal to the Bayes factor $B_{j i}$.

\section{B. Bayesian Model Averaging}

A natural strategy for model selection is to chose the most probable model $M_{j}$, namely the one with the highest posterior probability, $p\left(M_{j} \mid D\right)$. Alternatively, especially in cases where the posterior mass of the model space $\mathcal{M}$ is not concentrated only on one model, $M_{j}$, it is possible to consider averaging models using the posterior model probabilities as weights. Raftery, Madigan, and Hoeting (1997) show that BMA almost always improves on variable selection in terms of predictive performance.

Using Bayesian Model Averaging, inference for a quantity of interest $\Gamma$ can be constructed based on the posterior distribution

$$
p(\Gamma \mid D)=\sum_{j=1}^{K} p\left(\Gamma \mid D, M_{j}\right) p\left(M_{j} \mid D\right)
$$

which follows by the law of total probability. Therefore, the full posterior distribution of $\Gamma$ is a weighted average of the posterior distributions under each model $\left(M_{1}, \ldots, M_{K}\right)$, where the weights are the posterior model probabilities $p\left(M_{j} \mid D\right)$. The posterior model probabilities are obtained using (3). Using (5) one can compute the posterior mean and posterior variance for parameters $\theta_{l}$ as follows

$$
E\left(\theta_{l} \mid D\right)=\sum_{j=1}^{K} p\left(M_{j} \mid D\right) E\left(\theta_{l} \mid D, M_{j}\right)
$$

and

$$
\operatorname{Var}\left(\theta_{l} \mid D\right)=\sum_{j=1}^{K} p\left(M_{j} \mid D\right) \operatorname{Var}\left(\theta_{l} \mid D, M_{j}\right)+\sum_{j=1}^{K} p\left(M_{j} \mid D\right)\left[E\left(\theta_{l} \mid D, M_{j}\right)-E\left(\theta_{l} \mid D\right)\right]^{2} .
$$

The implementation of BMA presents a number of challenges, including the evaluation of the marginal probability in (4), the large number of possible models, and the specification of the prior model probabilities $p\left(M_{j}\right)$ as well as the parameters' prior, $p\left(\theta \mid M_{i}\right)$.

\footnotetext{
${ }^{7}$ As in Fernández, Ley and Steel (2001b).
} 


\section{Choice of Priors}

Evaluating Bayes factors required for hypothesis testing and Bayesian model selection or model averaging requires calculating the marginal likelihood

$$
p\left(D \mid M_{j}\right)=\int p\left(D \mid \theta, M_{j}\right) p\left(\theta \mid M_{j}\right) d \theta .
$$

Here, the dimension of the parameter $\theta$ is determined by model $M_{j}$. In many cases the likelihood $p\left(D \mid \theta, M_{i}\right)$ is fully specified with some nuisance parameter $\zeta$. Therefore we may write

$$
p\left(D \mid M_{i}\right)=\int p\left(D \mid \theta, \zeta, M_{i}\right) p\left(\theta, \zeta \mid M_{i}\right) d \theta d \zeta .
$$

In this case, determining the prior $p\left(\theta, \zeta \mid M_{i}\right)$ becomes an important issue. ${ }^{8}$

For Gaussian models the nuisance parameter is the variance $\sigma_{u}^{2}$ of the noise term. A common selection of the prior for the pair $\left(\theta, \sigma_{u}^{-2}\right)$ is its conjugate prior, Normal-Gamma distribution, which has the benefit of rendering a closed-form posterior ${ }^{9}$. With this prior $\theta$ is a Normal random variable with mean $\theta_{0}$ and variance $\sigma_{u}^{2} V$ given $\sigma_{u}^{2}$, while $\sigma_{u}^{-2}$ is a Gamma random variable with mean $\frac{\gamma}{\lambda}$ and variance $\frac{\gamma}{\lambda^{2}}$. Based on this prior, when (1) represents a Gaussian panel data model with fixed effects, the likelihood ratio of two different models, $M_{i}$ and $M_{j}$, becomes

$$
\begin{aligned}
\frac{p\left(D \mid M_{i}\right)}{p\left(D \mid M_{j}\right)} & =\left(\frac{\left|I+\tilde{Z}_{j}^{\prime} \tilde{Z}_{j} V_{j}\right|}{\left|I+\tilde{Z}_{i}^{\prime} \tilde{Z}_{i} V_{i}\right|}\right)^{1 / 2} \\
& \times\left(\frac{2 \lambda+S S E_{j}+\left(\hat{\theta}_{j}-\theta_{j}\right)^{\prime}\left(V_{j}+\left(\tilde{Z}_{j}^{\prime} \tilde{Z}_{j}\right)^{-1}\right)^{-1}\left(\hat{\theta}_{j}-\theta_{j}\right)}{2 \lambda+S S E_{i}+\left(\hat{\theta}_{i}-\theta_{i}\right)^{\prime}\left(V_{i}+\left(\tilde{Z}_{i}^{\prime} \tilde{Z}_{i}\right)^{-1}\right)^{-1}\left(\hat{\theta}_{i}-\theta_{i}\right)}\right)^{(\gamma+N(T-1)) / 2}
\end{aligned}
$$

where $\tilde{Z}$ stands for the demeaned values of $Z$. Due to the sensitivity of the Bayes factors to the prior parameters $\left\{\theta_{0}, V, \gamma, \lambda\right\}$, one often avoids choosing specific values for them, in order not to affect substantially the posterior distribution. As discussed in Kass and Wasserman (1995), and Fernández, Ley and Steel (2001a), one possibility is to use a diffuse

\footnotetext{
${ }^{8}$ Fernández, Ley and Steel (2001b) investigate a set of "benchmark" prior specifications in a linear regression context with model uncertainty in order to address the sensitivity of the posterior model probabilities to the specification of the priors.

${ }^{9}$ For a more detailed discussion see Kass and Wasserman (1995).
} 
prior for $\sigma_{u}$ with density $p\left(\sigma_{u}\right) \propto{\sigma_{u}}^{-1}$. This prior has a nice scale invariance property and is equivalent to setting $\gamma=\lambda=0$ in the Gamma distribution of $\sigma_{u}^{-2}$. For the prior distribution of $\theta$ conditioned on $\sigma_{u}^{-2}$, one popular choice is using Zellner's $g$-prior with 0 mean

$$
p\left(\theta \mid \sigma_{u}^{2}\right) \sim N\left(0, g^{-1}\left(\tilde{Z}^{\prime} \tilde{Z}\right)^{-1} \sigma_{u}^{2}\right)
$$

which can be motivated by the fact that the correlation of the OLS estimate $\hat{\theta}$ is proportional to $\left(\tilde{Z}^{\prime} \tilde{Z}\right)^{-1} \sigma_{u}^{2}$. This also leads to a simple likelihood ratio

$$
\frac{p\left(D \mid M_{i}\right)}{p\left(D \mid M_{j}\right)}=\frac{\left(1+g_{i}^{-1}\right)^{k_{1} / 2}}{\left(1+g_{j}^{-1}\right)^{k_{2} / 2}}\left(\frac{\frac{1}{g_{j}+1} S S E_{j}+\frac{g_{j}}{g_{j}+1} \tilde{y}^{\prime} \tilde{y}}{\frac{1}{g_{i}+1} S S E_{i}+\frac{g_{i}}{g_{i}+1} \tilde{y}^{\prime} \tilde{y}}\right)^{N(T-1) / 2}
$$

where $\tilde{y}$ stands for the demeaned values of $y$. The BACE procedure (proposed by Sala-iMartin, Doppelhofer, and Miller (2004)) is asymptotically equivalent to setting $g=N(T-1)$.

Alternatively, one can use what has been labeled in the literature as the BIC approach, where the likelihood ratio is approximated by

$$
\frac{p\left(D \mid M_{1}\right)}{p\left(D \mid M_{2}\right)} \sim \exp \left(-\frac{1}{2}\left(S S E_{1}-S S E_{2}\right)-\frac{1}{2}\left(k_{1}-k_{2}\right) \log (N(T-1))\right) .
$$

Here the approximation is $O_{p}\left(N^{-1 / 2}\right)$ when the implicit prior for $\theta$ is the unit information Normal prior as discussed in Kass and Wasserman (1995) and Kass and Raftery (1995).

Finally, several options exist for the specification of the model priors $p\left(M_{j}\right)$. For example, Fernández, Ley and Steel (2001b) assume a Uniform distribution over the model space, essentially implying that there is no preference for a specific model so $p\left(M_{1}\right)=p\left(M_{2}\right)=\ldots=p\left(M_{K}\right)=\frac{1}{K}$. Other options include penalizing models with more regressors. Sala-i-Martin, Doppelhofer and Miller (2004) use a prior model probability structure initially proposed by Mitchell and Beauchamp (1988). Assuming that each variable has an equal inclusion probability, the prior probability for model $M_{j}$ is

$$
p\left(M_{j}\right)=\left(\frac{\bar{k}}{k}\right)^{k_{j}}\left(1-\frac{\bar{k}}{k}\right)^{k-k_{j}}
$$

and the prior odds ratio is 


$$
\frac{p\left(M_{j}\right)}{p\left(M_{l}\right)}=\left(\frac{\bar{k}}{k}\right)^{k_{j}-k_{l}}\left(1-\frac{\bar{k}}{k}\right)^{k_{l}-k_{j}}
$$

where $k$ is the total number of regressors, $\bar{k}$ is the researcher's prior about the size of the model, $k_{j}$ is the number of included variables in model $M_{j}$, and $\frac{\bar{k}}{k}$ is the prior inclusion probability for each variable.

\section{Limited Information BAyesian Model Averaging}

This section provides a discussion of the LIBMA using a dynamic panel data model with endogenous and exogenous regressors and derives the limited information criterion using the moment conditions implied by the GMM framework.

\section{A. A Dynamic Panel Data Model with Endogenous Regressors}

Let us consider the case where a researcher is faced with model uncertainty when trying to estimate a dynamic model for panel data. We assume that the universe of potential explanatory variables, indexed by the set $\mathbf{U}$, consists of the lagged dependent variable, indexed by 1 , a set of $m$ exogenous variables, indexed by $\mathbf{X}$, as well as a set of $q$ endogenous variables, indexed by $\mathbf{W}$, such that $\{\{1\}, \mathbf{X}, \mathbf{W}\}$ is a partition of $\mathbf{U}$. Therefore, for a given model $M_{j} \subset \mathbf{U},(2)$ becomes

$$
\begin{aligned}
y_{i t} & =\left(\begin{array}{lll}
y_{i, t-1} & \mathbf{x}_{\mathbf{i t}} \quad \mathbf{w}_{\mathbf{i t}}
\end{array}\right) \mathbf{C}_{M_{j}}\left(\begin{array}{lll}
\alpha & \theta_{x} & \theta_{w}
\end{array}\right)^{\prime}+u_{i t} \\
u_{i t} & =\eta_{i}+v_{i t} \\
|\alpha| & <1 ; i=1,2, \ldots, N ; \quad t=1,2, \ldots, T .
\end{aligned}
$$

Here $y_{i t}, \mathbf{x}_{\mathbf{i t}}$ and $\mathbf{w}_{\mathbf{i t}}$ are observed variables, $\eta_{i}$ is the unobserved individual effect while $v_{i t}$ is the idiosyncratic random error. The exact distributions for $v_{i t}$ and $\eta_{i}$ are not specified here, but assumptions about some of their moments and correlation with the regressors are made explicit below. It is assumed that $E\left(v_{i t}\right)=0$ and that $v_{i t}$ 's are not serially correlated. $\mathbf{x}_{i t}$ is a $1 \times m$ vector of exogenous variables while $\mathbf{w}_{i t}$ is a $1 \times q$ vector of endogenous variables. Therefore the total number of possible explanatory variables is $k=m+q+1$. The observed variables span $N$ individuals and $T$ periods, where $T$ is small relative to $N$. The unknown parameters $\alpha, \theta_{x}$, and $\theta_{w}$ are to be estimated. In this model, $\alpha$ is a scalar, $\theta_{x}$ is a $1 \times m$ vector while $\theta_{w}$ is a $1 \times q$ vector. 
Given the assumptions made so far, for any model $M_{j}$, and any set of exogenous variables, $\mathbf{x}_{i t}$, we have

$$
E\left(x_{i t}^{l} v_{i s}\right)=0, \quad \forall i, t, s ; \quad x_{i t}^{l} \in \mathbf{x}_{i t} .
$$

Similarly, for any endogenous variable we have

$$
E\left(w_{i t}^{l} v_{i s}\right)\left\{\begin{array}{l}
\neq 0, \quad s \leq t \\
=0, \text { otherwise }
\end{array}, \quad w_{i t}^{l} \in \mathbf{w}_{i t} .\right.
$$

Note that, in principle, the correlations between endogenous variables and the idiosyncratic error may change over different individuals and/or periods.

\section{B. Estimation and Moment Conditions}

A common approach for estimating the model (12) is to use the system GMM framework developed by Blundell and Bond (1998). This implies constructing the instruments set and moment conditions for the "level equations" (12) and combining them with the moment conditions using the instruments corresponding to the first differences equations. The first differences (FD) equations corresponding to model (12) are given by

$$
\begin{aligned}
& \Delta y_{i t}=\left(\begin{array}{lll}
\Delta y_{i, t-1} \quad \Delta \mathbf{x}_{\mathbf{i t}} \quad \Delta \mathbf{w}_{\mathrm{it}}
\end{array}\right) \mathbf{C}_{M_{j}}\left(\begin{array}{lll}
\alpha & \theta_{x} & \theta_{w}
\end{array}\right)^{\prime}+\Delta v_{i t} \\
& |\alpha|<1 ; i=1,2, \ldots, N ; \quad t=2,3, \ldots, T .
\end{aligned}
$$

One assumption required for the FD equations is that the initial value of $\mathbf{y}, y_{i 0}$, is predetermined, that is, $E\left(y_{i 0} v_{i s}\right)=0$ for $s=2,3, \ldots, T$. Since $y_{i, t-2}$ is not correlated with $\Delta v_{i t}$ we can use it as an instrument. Hence we have $E\left(y_{i, t-2} \Delta v_{i t}\right) \neq 0$ for $t=2,3, \ldots, T$. Moreover, $y_{i, t-3}$ is also not correlated with $\Delta v_{i t}$. Therefore, as long as we have enough observations, that is $T \geq 3, y_{i, t-3}$ can be used as an instrument. Assuming that we have more than two observations in the time dimension, the following moment conditions could be used for estimation

$$
E\left(y_{i, t-s} \Delta v_{i t}\right)=0, \quad t=2,3, \ldots, T ; \quad s=2,3, \ldots, t ; \text { for } T \geq 2, \quad i=1,2, \ldots, N .
$$


Similarly, the first difference of the exogenous variable $\Delta x_{i t}^{l}, x_{i t}^{l} \in \mathbf{x}_{i t}$ is not correlated with $\Delta v_{i t}$ and therefore we can use it as an instrument. ${ }^{10}$ That gives us additional moment conditions

$$
E\left(\Delta x_{i t}^{l} \Delta v_{i t}\right)=0, \quad t=2,3, \ldots, T ; \quad l=1, \ldots, m ; \quad i=1,2, \ldots, N
$$

The endogenous variable $w_{i, t-2}^{l}, \quad w_{i, t-2}^{l} \in \mathbf{w}_{i t}$, is not correlated with $\Delta v_{i t}$ and therefore it can be used as an instrument. We have the following possible moment conditions

$$
\begin{array}{r}
E\left(w_{i, t-s}^{l} \Delta v_{i t}\right)=0, \quad t=3,4, \ldots, T ; \quad s=2, \ldots t-1 \\
\text { for } T \geq 3, \quad l=1,2, \ldots, q ; \quad i=1, \ldots, N .
\end{array}
$$

Table 1 summarizes the moment conditions that could be used for the FD equation.

Table 1. Moment Conditions for the FD Equation

\begin{tabular}{|c|l|l|}
\hline & Instruments & Moment conditions \\
\hline $\begin{array}{c}\text { Lagged dependent variable } \\
\Delta y_{i, t-1}\end{array}$ & $y_{i, t-2}, \ldots, y_{i 0}$ & $E\left(y_{i, t-s} \Delta v_{i t}\right)=0, \quad t=2,3, \ldots T ; s=2,3, \ldots, t$ \\
\hline Exogenous variable $\Delta x_{i t}^{l}$ & $x_{i t}^{l}, \ldots, x_{i 1}^{l}$ & $E\left(\Delta x_{i t}^{l} \Delta v_{i t}\right)=0, \quad t=2,3, \ldots, T ; \quad l=1, \ldots, m$ \\
\hline Endogenous variable $\Delta w_{i t}^{l}$ & $w_{i, t-2}^{l}, \ldots, w_{i, 1}^{l}$ & $\begin{array}{c}E\left(w_{i, t-s}^{l} \Delta v_{i t}\right)=0, \quad t=3,4, \ldots, T ; \\
s=2,3, \ldots t-1 ; 1=1,2, \ldots, q\end{array}$ \\
& & \\
\hline
\end{tabular}

The FD equation provides $T(T-1) / 2$ moment conditions for the lagged dependent variable, $m(T-1)$ moment conditions for the exogenous variables, and $q(T-2)(T-1) / 2$ moment conditions for the endogenous variables.

Going back to the equation in levels (12), it is easy to see that first differences for the lagged dependent variable are not correlated with either the individual effects or the idiosyncratic error term and hence we can use the following moment conditions

$$
E\left(\Delta y_{i, t-1} u_{i t}\right)=0, \quad t=2,3, \ldots, T .
$$

\footnotetext{
${ }^{10}$ It is common in the literature to use $x_{i t}^{l} \in \mathbf{x}_{i t}$ as an instrument, instead of $\Delta x_{i t}^{l}$. Then the moment condition (17) becomes $E\left(x_{i t}^{l} \Delta v_{i t}\right)=0$.
} 
Similarly, for the endogenous variables the first difference $\Delta w_{i, t-1}^{l}$ is not correlated with $u_{i t}$. Therefore, assuming that $w_{i, 0}^{l}$ is observable, and as long as $T \geq 3$ we have the following additional moment conditions

$$
E\left(\Delta w_{i, t-1}^{l} u_{i t}\right)=0, \quad t=3,4, \ldots, T ; \quad l=1,2, \ldots, q .
$$

Finally, based on the assumptions made so far, the exogenous variables $x_{i t}^{l} \in \mathbf{x}_{i t}$ are not correlated with current realizations of $u_{i t}$ and hence one can use another set of moment conditions $^{11}$

$$
E\left(x_{i t}^{l} u_{i t}\right)=0, \quad t=1,2, \ldots, T ; \quad l=1,2, \ldots, m .
$$

Table 2 summarizes the moment conditions for the level equation.

Table 2. Moment Conditions for the Level Equation

\begin{tabular}{|l|l|l|}
\hline & Instruments & Moments \\
\hline $\begin{array}{c}\text { Lagged dependent variable } \\
y_{i, t-1}\end{array}$ & $\Delta y_{i, t-1}$ & $E\left(\Delta y_{i, t-1} u_{i t}\right)=0, \quad t=2,3, \ldots, T$ \\
\hline Exogenous variable $x_{i t}^{l}$ & $x_{i t}^{l}$ & $E\left(x_{i t}^{l} u_{i t}\right)=0, \quad t=1,2, \ldots, T ; \quad \mathrm{l}=1,2, \ldots, m$ \\
\hline Endogenous variable $w_{i t}^{l}$ & $\Delta w_{i, t-1}^{l}$ & $E\left(\Delta w_{i, t-1}^{l} u_{i t}\right)=0, \quad t=3,4, \ldots, T ; \quad l=1,2, \ldots, q$ \\
\hline
\end{tabular}

The equation in levels provides $(T-1)$ moment conditions for the lagged dependent variable, $m T$ moment conditions for the exogenous variables, and $q(T-2)$ moment conditions for the endogenous variables.

We group the moment conditions into matrices the following way. Let $Y_{i}$ be the $(T-1) \times T(T-1) / 2$ matrix of lagged dependent variable used as instruments for the FD equation

${ }^{11}$ The implicit assumption made here is that the exogenous variables are not correlated with the individual effect. If that assumption can not be made, $\Delta x_{i t}^{l}$ should be used as instruments, giving us the following moment conditions $E\left(\Delta x_{i t}^{l} u_{i t}\right)=0, t=1,2, \ldots, T ; 1=1,2, \ldots, m$. 


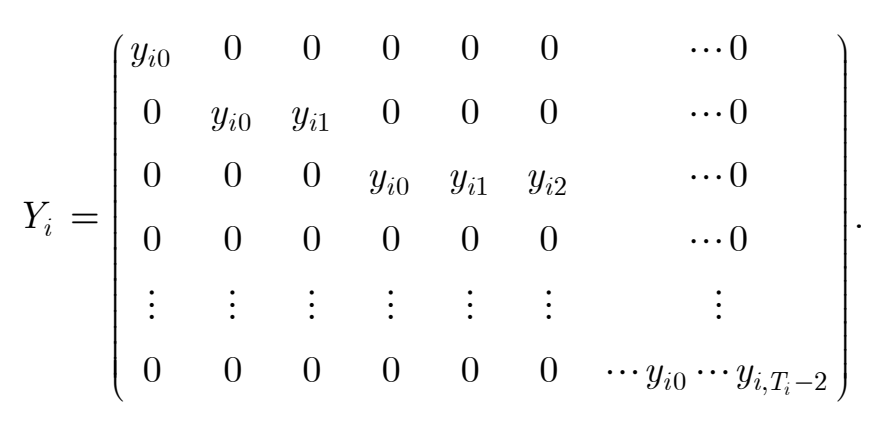

Similarly, $W_{i}$ denotes the $(T-1) \times q(T-2)(T-1) / 2$ matrix of endogenous variables

$$
W_{i}=\left(\begin{array}{lllllll}
0 & 0 & 0 & \cdots & 0 & \cdots 0 & 0 \\
w_{i 1}^{1} & 0 & 0 & \cdots & w_{i 1}^{q} & \cdots 0 & 0 \\
0 & w_{i 1}^{1} & w_{i 2}^{1} & \cdots & 0 & \cdots 0 & 0 \\
0 & 0 & 0 & \cdots & 0 & \cdots 0 & 0 \\
\vdots & \vdots & \vdots & \vdots & \vdots & \vdots & \vdots \\
0 & 0 & 0 & \cdots & 0 & \cdots w_{i, T-3}^{q} & w_{i, T-2}^{q}
\end{array}\right) .
$$

For the level equation we have the $T \times(T-1)$ instruments matrix $D Y_{i}$ consisting of first differences of the dependent variable and the $T \times q(T-2)$ instruments matrix $D W_{i}$ consisting of first differences of the endogenous variables

$$
D Y_{i}=\left(\begin{array}{lllll}
0 & 0 & 0 & \cdots & 0 \\
\Delta y_{i 1} & 0 & 0 & \cdots & 0 \\
0 & \Delta y_{i 2} & 0 & \cdots & 0 \\
0 & 0 & \Delta y_{i 3} & \cdots & 0 \\
\vdots & \vdots & \vdots & \cdots & \vdots \\
0 & 0 & 0 & \cdots & \Delta y_{i, T-1}
\end{array}\right) \quad D W_{i}=\left(\begin{array}{lll}
0 & 0 \cdots & 0 \\
0 \cdots & 0 \cdots & 0 \\
\Delta w_{i 2}^{1} \cdots & \Delta w_{i 2}^{q} \cdots & 0 \\
0 \cdots & 0 \cdots & 0 \\
\vdots & \cdots & \vdots \\
0 \cdots & 0 \cdots & \Delta w_{i, T-1}^{q}
\end{array}\right)
$$

Further let $X_{i}$ and $D X_{i}$ denote the following $T \times m$ and $(T-1) \times m$ matrices of exogenous and first differenced exogenous variables, respectively

$$
D X_{i}=\left(\begin{array}{lllll}
\Delta x_{i 2}^{1} & \Delta x_{i 2}^{2} & \Delta x_{i 2}^{3} & \cdots & \Delta x_{i 2}^{m} \\
\Delta x_{i 3}^{1} & \Delta x_{i 3}^{2} & \Delta x_{i 3}^{3} & \cdots & \Delta x_{i 3}^{m} \\
\Delta x_{i 4}^{1} & \Delta x_{i 4}^{2} & \Delta x_{i 4}^{3} & \cdots & \Delta x_{i 4}^{m} \\
\vdots & \vdots & \vdots & \cdots & \vdots \\
\Delta x_{i T}^{1} & \Delta x_{i T}^{2} & \Delta x_{i T}^{3} & \cdots & \Delta x_{i T}^{m}
\end{array}\right) \quad X_{i}=\left(\begin{array}{lllll}
x_{i 1}^{1} & x_{i 1}^{2} & x_{i 1}^{3} & \cdots & x_{i 1}^{m} \\
x_{i 2}^{1} & x_{i 2}^{2} & x_{i 2}^{3} & \cdots & x_{i 2}^{m} \\
x_{i 3}^{1} & x_{i 3}^{2} & x_{i 3}^{3} & \cdots & x_{i 3}^{m} \\
\vdots & \vdots & \vdots & \cdots & \vdots \\
x_{i T}^{1} & x_{i T}^{2} & x_{i T}^{3} & \cdots & x_{i T}^{m}
\end{array}\right) .
$$


For the exogenous variables, we aggregate the moment conditions across all periods from both the first difference equation and the level equation. Thus, we are left with one moment condition for each of the exogenous variables

$$
\sum_{t=2}^{T} E\left(\Delta x_{i t}^{l} \Delta v_{i t}\right)+\sum_{t=1}^{T} E\left(x_{i t}^{l} u_{i t}\right)=0, \quad 1=1, \ldots, m ; \quad i=1,2, \ldots, N .
$$

Let $u_{i}$ and $D v_{i}$ denote the $T \times 1$ and $(T-1) \times 1$ matrices of the error term and the first differenced idiosyncratic random error, respectively, as defined in model (12).

$$
u_{i}=\left(\begin{array}{llll}
u_{i 1} & u_{i 2} & \cdots & u_{i T}
\end{array}\right)^{\prime} \quad \quad D v_{i}=\left(\begin{array}{llll}
\Delta v_{i 2} & \Delta v_{i 3} & \cdots & \Delta v_{i T}
\end{array}\right)^{\prime} .
$$

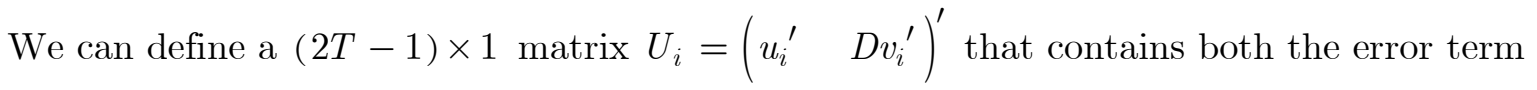
and the first differenced idiosyncratic random error. The moment conditions can now be written in matrix form

$$
E\left[G^{\prime}{ }_{i} U_{i}\right]=0
$$

where $G_{i}$ is a $(2 T-1) \times(m-1+(T+1)((T-2) q+T) / 2)$ matrix defined as

$$
G_{i}=\left(\begin{array}{lllll}
X_{i} & D Y_{i} & 0_{T \times T(T-1) / 2} & D W_{i} & 0_{T \times q(T-1)(T-2) / 2} \\
D X_{i} & 0_{(T-1) \times(T-1)} & Y_{i} & 0_{(T-1) \times q(T-2)} & W_{i}
\end{array}\right) .
$$

Based on the moment conditions (27) we propose a limited information criterion that can be used in Bayesian model selection and averaging. In the next section we provide details on how to construct this criterion.

\section{The Limited Information Criterion}

As we pointed out in section II.C, evaluating the Bayes factors needed for hypothesis testing and Bayesian model selection or model averaging requires calculating the marginal likelihood

$$
p\left(D \mid M_{j}\right)=\int p\left(D \mid \theta, M_{j}\right) p\left(\theta \mid M_{j}\right) d \theta .
$$

Given that we choose to use the Generalized Method of Moments (GMM) for estimating the parameters of the model, the assumptions we have made so far do not give us a fully specified parametric likelihood $p\left(D \mid \theta, M_{i}\right)$. Therefore, we have to build the model likelihood in a fashion consistent with the Bayesian paradigm using the information provided by the moment conditions. 
The construction of non-parametric likelihood functions has received lately a good deal of attention in the literature. Several approaches have been used to derive or estimate non-parametric likelihood functions. For example, Back and Brown (1993) provide a method of estimating a distribution function using only information derived from moment restrictions. Kim (2002) uses information projection onto a family of probability measures and constructs the likelihood by using a transformation of the GMM objective function. Hong and Preston (2008) build a quasi likelihood which is based on objective functions used for extremum estimation (see also Chernozhukov and Hong (2003)). Schennach (2005) builds a likelihood function that is the nonparametric limit result of a formal Bayesian procedure where the prior for the data favors distributions with a large entropy. Further the prior is conditioned on the moment equations. In this fashion it becomes feasible to compute a likelihood function that is closely related to empirical likelihood. Finally, Ragusa (2008) projects a reference distribution onto the space of distributions that are consistent with a set of moment restrictions and obtains the likelihood by integrating out the nuisance parameters.

In this section we propose a method of constructing the model likelihoods and posteriors based only on the information elicited from the moment conditions (27). Suppose we have a strictly stationary and ergodic random process $\left\{\xi_{i}\right\}_{i=1}^{\infty}$, which takes value in the space $\Xi$, and a parameter space $\Theta \subset R^{k}$. Then there exists a function $g: \Xi \times \Theta \rightarrow R^{l}$ which satisfies the following conditions

(1) it is continuous on $\Theta$;

(2) $E\left[g\left(\xi_{i}, \theta\right)\right]$ exists and is finite for every $\theta \in \Theta$; and

(3) $E\left[g\left(\xi_{i}, \theta\right)\right]$ is continuous on $\theta$.

We further assume that the moment conditions, $E\left[g\left(\xi_{i}, \theta\right)\right]=0$, hold for a unique unknown $\theta_{0} \in \Theta$. Let $\hat{g}_{N}(\theta)=N^{-1} \sum_{i=1}^{N} g\left(\xi_{i}, \theta\right)$ denote the sample mean of the moment conditions, and assume that $E\left[g\left(\xi_{i}, \theta_{0}\right) g^{\prime}\left(\xi_{i}, \theta_{0}\right)\right]$ and $S\left(\theta_{0}\right) \equiv \lim _{n \rightarrow \infty} \operatorname{Var}\left[N^{1 / 2} \hat{g}_{N}\left(\theta_{0}\right)\right]$ exist and are finite positive definite matrices. Then the following standard result holds (for a proof see Hall (2005) Lemma 3.2).

Lemma 1. Under the above assumptions, $N^{1 / 2} \hat{g}_{N}\left(\theta_{0}\right) \stackrel{d}{\longrightarrow} N\left(0, S\left(\theta_{0}\right)\right)$. 
That is, the random vector $N^{1 / 2} \hat{g}_{N}\left(\theta_{0}\right)$ convergences in distribution to a multivariate Normal distribution.

For model (12), the moment conditions for individual $i$ discussed in the previous section can be written in the following form

$$
g\left(\xi_{i}, \theta\right)=G_{i}^{\prime}\left(\tilde{\mathbf{y}}_{\mathbf{i}}-\tilde{\mathbf{z}}_{\mathbf{i}} \theta\right)
$$

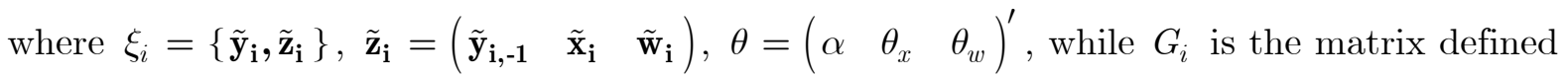
in (28). The vectors $\tilde{\mathbf{y}}_{\mathbf{i}}$ and $\tilde{\mathbf{y}}_{\mathbf{i},-1}$ for the dependent variable and the lagged dependent variable, respectively, are defined as follows

$$
\begin{aligned}
& \tilde{\mathbf{y}}_{\mathbf{i}}=\left(\begin{array}{llllllll}
y_{i 1} & y_{i 2} & \cdots & y_{i T} & \Delta y_{i 2} & \Delta y_{i 3} & \cdots & \Delta y_{i T}
\end{array}\right)^{\prime} \\
& \tilde{\mathbf{y}}_{\mathbf{i},-1}=\left(\begin{array}{llllllll}
y_{i 0} & y_{i 1} & \cdots & y_{i, T-1} & \Delta y_{i 1} & \Delta y_{i 2} & \cdots & \Delta y_{i, T-1}
\end{array}\right)^{\prime} .
\end{aligned}
$$

The matrix $\tilde{\mathbf{x}}_{\mathbf{i}}$ for the exogenous variables is given by

$$
\tilde{\mathbf{x}}_{i}=\left(\begin{array}{lllll}
x_{i 1}^{1} & x_{i 1}^{2} & x_{i 1}^{3} & \cdots & x_{i 1}^{m} \\
\vdots & \vdots & \vdots & \cdots & \vdots \\
x_{i T}^{1} & x_{i T}^{2} & x_{i T}^{3} & \cdots & x_{i T}^{m} \\
\Delta x_{i 2}^{1} & \Delta x_{i 2}^{2} & \Delta x_{i 2}^{3} & \cdots & \Delta x_{i 2}^{m} \\
\vdots & \vdots & \vdots & \vdots & \vdots \\
\Delta x_{i T}^{1} & \Delta x_{i T}^{2} & \Delta x_{i T}^{3} & \cdots & \Delta x_{i T}^{m}
\end{array}\right)
$$

while the matrix $\tilde{\mathbf{w}}_{\mathbf{i}}$ for the endogenous variables is defined as follows

$$
\tilde{\mathbf{w}}_{i}=\left(\begin{array}{lllll}
w_{i 1}^{1} & w_{i 1}^{2} & w_{i 1}^{3} & \cdots & w_{i 1}^{q} \\
\vdots & \vdots & \vdots & \cdots & \vdots \\
w_{i T}^{1} & w_{i T}^{2} & w_{i T}^{3} & \cdots & w_{i T}^{q} \\
\Delta w_{i 2}^{1} & \Delta w_{i 2}^{2} & \Delta w_{i 2}^{3} & \cdots & \Delta w_{i 2}^{q} \\
\vdots & \vdots & \vdots & \vdots & \vdots \\
\Delta w_{i T}^{1} & \Delta w_{i T}^{2} & \Delta w_{i T}^{3} & \cdots & \Delta w_{i T}^{q}
\end{array}\right) .
$$

Therefore $\hat{g}_{N}\left(\theta_{0}\right)=N^{-1} \sum_{i=1}^{N} G_{i}^{\prime} \tilde{\mathbf{y}}_{\mathbf{i}}-N^{-1} \sum_{i=1}^{N} G_{i}^{\prime} \tilde{\mathbf{z}}_{\mathbf{i}} \theta_{0}$. By Lemma 1, one may write the likelihood for $\theta$ as

$$
p\left(N^{-1} \sum_{i=1}^{N} G_{i}^{\prime} \tilde{\mathbf{y}}_{\mathbf{i}} \mid \theta, N^{-1} \sum_{i=1}^{N} G_{i}^{\prime} \tilde{\mathbf{z}}_{\mathbf{i}}\right) \propto \exp \left(-\frac{1}{2} N \hat{g}_{N}^{\prime}(\theta) S_{(\theta)}^{-1} \hat{g}_{N}(\theta)\right) .
$$


Hence, the model likelihood can be expressed as

$$
\int_{\Theta} p\left(N^{-1} \sum_{i=1}^{N} G_{i}^{\prime} \tilde{\mathbf{y}}_{\mathbf{i}} \mid \theta\right) p(\theta) d \theta \propto \int_{\Theta} \exp \left(-\frac{1}{2} N \hat{g}_{N}^{\prime}(\theta) S_{(\theta)}^{-1} \hat{g}_{N}(\theta)\right) p(\theta) d \theta .
$$

Assuming that the prior $p(\theta)$ is second order differentiable around $\hat{\theta}_{0}$ and using the Laplace approximation, we obtain that the model likelihood is proportional to

$$
\begin{array}{r}
\int_{\Theta} p\left(N^{-1} \sum_{i=1}^{N} G_{i}^{\prime} \tilde{\mathbf{y}}_{\mathbf{i}} \mid \theta\right) p(\theta) d \theta \propto \exp \left(-\frac{1}{2} N \hat{g}_{N}^{\prime}\left(\hat{\theta}_{0}\right) S_{\left(\hat{\theta}_{0}\right)}^{-1} \hat{g}_{N}\left(\hat{\theta}_{0}\right)+\log p\left(\hat{\theta}_{0}\right)+\frac{k}{2} \log 2 \pi-\right. \\
\left.\frac{1}{2} \log \operatorname{det} \frac{\partial^{2}}{\partial \theta^{2}}\left(\frac{1}{2} N \hat{g}_{N}^{\prime}\left(\hat{\theta}_{0}\right) S_{\left(\hat{\theta}_{0}\right)}^{-1} \hat{g}_{N}\left(\hat{\theta}_{0}\right)\right)\right)
\end{array}
$$

where $\hat{\theta}_{0} \equiv \arg \min _{\theta} N \hat{g}_{N}^{\prime}(\theta) S_{(\theta)}^{-1} \hat{g}_{N}(\theta)$ is the GMM estimate of $\theta_{0}$ with weighting matrix $S_{(\theta)}^{-1}$. Noting the fact that $\partial^{2}\left(\hat{g}_{N}^{\prime} S^{-1} \hat{g}_{N}\right) /\left.\partial \theta^{2}\right|_{\theta=\hat{\theta}_{0}}$ is a $k \times k$ matrix of order $O_{p}(1)$ due to the ergodicity assumption, the model likelihood can be approximated by

$$
\int_{\Theta} p\left(N^{-1} \sum_{i=1}^{N} G_{i}^{\prime} \tilde{\mathbf{y}}_{\mathbf{i}} \mid \theta\right) p(\theta) d \theta \propto \exp \left(-\frac{1}{2} N \hat{g}_{N}^{\prime}\left(\hat{\theta}_{0}\right) S_{\left(\hat{\theta}_{0}\right)}^{-1} \hat{g}_{N}\left(\hat{\theta}_{0}\right)-\frac{k}{2} \log N\right)
$$

where $k$ is the dimension of vector $\theta$. Alternatively, the above approximation has the order of $O_{p}\left(N^{-1 / 2}\right)$ if the unit information prior for $\theta$ is used with $\partial^{2}\left(\hat{g}_{N}^{\prime} S^{-1} \hat{g}_{N}\right) /\left.\partial \theta^{2}\right|_{\theta=\hat{\theta}_{0}}$ as its variance-covariance matrix, that is, the prior distribution for $\theta, p(\theta)$, is given by $N\left(0, \partial^{2}\left(\hat{g}_{N}^{\prime} S^{-1} \hat{g}_{N}\right) /\left.\partial \theta^{2}\right|_{\theta=\hat{\theta}_{0}}\right)$.

For a given model $M_{j}$ for which $\theta$ has $k_{j}$ elements different from zero, with the estimate denoted by $\hat{\theta}_{0, j}$, the model likelihood (35) becomes

$$
\int_{\Theta} p\left(N^{-1} \sum_{i=1}^{N} G_{i}^{\prime} \tilde{\mathbf{y}}_{\mathbf{i}} \mid \theta, M_{j}\right) p(\theta) d \theta \propto \exp \left(-\frac{1}{2} N \hat{g}_{N}^{\prime}\left(\hat{\theta}_{0, j}\right) S_{\left(\hat{\theta}_{0, j}\right.}^{-1} \hat{g}_{N}\left(\hat{\theta}_{0, j}\right)-\frac{k_{j}}{2} \log N\right) \cdot(36
$$

Then the moment conditions (27) associated with model $M_{j}$ can be written as $E\left[G_{i}{ }^{\prime}\left(\tilde{\mathbf{y}}_{\mathbf{i}}-\tilde{\mathbf{z}}_{\mathbf{i}} C_{M_{j}} \theta_{0}\right)\right]=0$ where $C_{M_{j}}$ is a diagonal choice matrix such that its diagonal will have 1's if the corresponding variable is included in the model and 0's otherwise. Recognizing that the estimate $\hat{\theta}_{0}$ differs from model to model, the sample mean of the moment conditions for model $M_{j}$ can be written as $\hat{g}_{N}\left(\hat{\theta}_{0}\right)=N^{-1} \sum_{i=1}^{N} G_{i}^{\prime}\left(\tilde{\mathbf{y}}_{\mathbf{i}}-\tilde{\mathbf{z}}_{\mathbf{i}} C_{M_{j}} \hat{\theta}_{0, j}\right)$.

It is easy to see that $G_{i}{ }^{\prime}, \tilde{\mathbf{y}}_{\mathbf{i}}$, and $\tilde{\mathbf{z}}_{\mathbf{i}}$ are the same across all models. In other words, the 
moment conditions and the observable data are the same across the universe of models. ${ }^{12}$ allowing us to make valid comparisons of posterior probabilities, in accordance to the principle of Bayesian factor analysis. Therefore, by using (36), one can compute the posterior odds ratio of two models $M_{1}$ and $M_{2}$ by

$$
\begin{aligned}
& \frac{p\left(M_{1} \mid N^{-1} \sum_{i=1}^{N} G_{i}^{\prime} \tilde{\mathbf{y}}_{i}\right)}{p\left(M_{2} \mid N^{-1} \sum_{i=1}^{N} G_{i}^{\prime} \tilde{\mathbf{y}}_{i}\right)}=\frac{p\left(M_{1}\right)}{p\left(M_{2}\right)} \frac{p\left(N^{-1} \sum_{i=1}^{N} G_{i}^{\prime} \tilde{\mathbf{y}}_{i} \mid M_{1}\right)}{p\left(N^{-1} \sum_{i=1}^{N} G_{i}^{\prime} \tilde{\mathbf{y}}_{i} \mid M_{2}\right)} \\
&=\frac{p\left(M_{1}\right)}{p\left(M_{2}\right)} \exp \left(-\frac{1}{2}\left(N \hat{g}_{N}^{\prime}\left(\hat{\theta}_{0,1}\right) S_{\left(\hat{\theta}_{0,1}\right)}^{-1} \hat{g}_{N}\left(\hat{\theta}_{0,1}\right)-N \hat{g}_{N}^{\prime}\left(\hat{\theta}_{0,2}\right) S_{\left(\hat{\theta}_{0,2}\right)}^{-1} \hat{g}_{N}\left(\hat{\theta}_{0,2}\right)\right)-\right. \\
&\left.\left(\frac{k_{1}-k_{2}}{2} \log N\right)\right),
\end{aligned}
$$

which has the same form of BIC as fully specified models. We use iterative GMM estimation with moment conditions $E\left[G_{i}^{\prime}\left(\tilde{\mathbf{y}}_{\mathbf{i}}-\tilde{\mathbf{z}}_{\mathbf{i}} C_{M_{j}} \theta_{0, j}\right)\right]=0$ to approximate the Bayesian factors above. A consistent estimate of the weighting matrix is used to replace $S_{\left(\hat{\theta}_{0}\right)}^{-1}$ in $(37)$.

\section{Monte Carlo Simulation And Results}

In this section we describe the Monte Carlo simulations intended to assess the performance of LIBMA. We compute posterior model probabilities, inclusion probabilities for each variable in the universe considered, and parameter statistics. These statistics provide a description of how well our procedure helps the inference process both in a Bayesian model selection and a Bayesian model averaging framework.

\section{A. The Data Generating Process}

We consider the case where the universe of potential explanatory variables contains 6 exogenous variables, 2 endogenous variables and the lagged dependent variable. Throughout our simulations we maintain the number of periods constant, that is, $T=4$ and we vary the number of individuals, $N$.

\footnotetext{
${ }^{12}$ This approach is in line with the model selection procedure proposed by Andrews and Lu (2001).
} 
For every individual $i$ and period $t$, the first four exogenous variables are generated as follows

$$
\begin{aligned}
& \left(\begin{array}{llll}
x_{i t}^{1} & x_{i t}^{2} & x_{i t}^{3} & x_{i t}^{4}
\end{array}\right)=\left(\begin{array}{llll}
0.3 & 0.4 & 0.8 & 0.5
\end{array}\right)+\mathbf{r}_{\mathbf{t}} \\
& \text { with } \quad \mathbf{r}_{\mathbf{t}} \sim N\left(0, I_{4}\right) \text { for } t=0,1, \ldots, T ; \quad i=1, \ldots, N,
\end{aligned}
$$

where $I_{4}$ is the four dimensional identity matrix. We allow for some correlation between the first two and the last two exogenous variables. That is, $\left(\begin{array}{ll}\mathbf{x}_{\mathbf{i}}^{\mathbf{5}} & \mathbf{x}_{\mathbf{i}}^{\mathbf{6}}\end{array}\right)$ are correlated with $\left(\begin{array}{ll}\mathbf{x}_{\mathbf{i}}^{1} & \mathbf{x}_{\mathbf{i}}^{2}\end{array}\right)$ such that for every individual $i$ and period $t$, the data generating process is given by

$$
\begin{aligned}
& \left(\begin{array}{ll}
x_{i t}^{5} & x_{i t}^{6}
\end{array}\right)=\left(\left(\begin{array}{ll}
x_{i t}^{1} & x_{i t}^{2}
\end{array}\right)-\left(\begin{array}{ll}
0.3 & 0.4
\end{array}\right)\right) \cdot 0.1 \cdot\left(\begin{array}{ll}
1 & 2
\end{array}\right)^{\prime}\left(\begin{array}{ll}
1 & 1
\end{array}\right)+\left(\begin{array}{ll}
1.5 & 1.8
\end{array}\right)+\mathbf{r}_{t} \\
& \text { with } \mathbf{r}_{\mathbf{t}} \sim N\left(0, I_{2}\right) \text { for } t=0,1, \ldots, T ; \quad i=1, \ldots, N
\end{aligned}
$$

where $I_{2}$ is the two dimensional identity matrix.

Similarly, for the endogenous variables, $\left(\begin{array}{ll}\mathbf{w}_{\mathbf{i}}^{1} & \mathbf{w}_{\mathbf{i}}^{2}\end{array}\right)$, we have the following data generating process

$$
\begin{aligned}
& \left(\begin{array}{ll}
w_{i t}^{1} & w_{i t}^{2}
\end{array}\right)=0.9\left(\begin{array}{ll}
w_{i, t-1}^{1} & w_{i, t-1}^{2}
\end{array}\right)+10 v_{i t}\left(\begin{array}{ll}
1 & 1
\end{array}\right)+\mathbf{r}_{\mathbf{t}} \text { for } t=1,2, \ldots, T \\
& \left(\begin{array}{ll}
w_{i 0}^{1} & w_{i 0}^{2}
\end{array}\right)=10 v_{i 0}\left(\begin{array}{ll}
1 & 1
\end{array}\right)+\mathbf{r}_{\mathbf{0}} \\
& \text { with } v_{i t} \sim N\left(0, \sigma_{v}^{2}\right) \text { and } \mathbf{r}_{\mathbf{t}} \sim N\left(0, I_{2}\right) \text { for } t=0,1, \ldots, T .
\end{aligned}
$$

As the data generating process for the endogenous variables indicates, the overall error term $v_{i t}$ is assumed to be distributed normally here. We relax the normality assumption later.

For $t=0$, the dependent variable is generated by

$$
\begin{aligned}
& y_{i 0}=\frac{1}{(1-\alpha)}\left(\mathbf{x}_{i 0} \theta_{x}+\mathbf{w}_{i 0} \theta_{w}+\eta_{i}+v_{i 0}\right) \\
& \text { with } v_{i 0} \sim N\left(0, \sigma_{v}^{2}\right) \text { and } \eta_{i} \sim N\left(0, \sigma_{\eta}^{2}\right)
\end{aligned}
$$

where $\quad \theta_{x}=\left(\begin{array}{llllll}0.07 & 0 & 0 & -0.09 & 0 & 0.1\end{array}\right)^{\prime}, \quad \theta_{w}=\left(\begin{array}{ll}0 & -0.1\end{array}\right)^{\prime}, \quad \mathbf{w}_{i 0}=\left(\begin{array}{ll}w_{i 0}^{1} & w_{i 0}^{2}\end{array}\right), \quad$ and $x_{i 0}=\left(\begin{array}{llllll}x_{i 0}^{1} & x_{i 0}^{2} & x_{i 0}^{3} & x_{i 0}^{4} & x_{i 0}^{5} & x_{i 0}^{6}\end{array}\right)$.

For $t=1,2, \ldots, T$ the data generating process is given by

$$
\begin{aligned}
& y_{i t}=\alpha y_{i, t-1}+\theta_{x} \mathbf{x}_{\mathbf{i t}}+\theta_{w} \mathbf{w}_{\mathbf{i t}}+\eta_{i}+v_{i t} \\
& \text { with } v_{i t} \sim N\left(0, \sigma_{v}^{2}\right) \text { and } \eta_{i} \sim N\left(0, \sigma_{\eta}^{2}\right) .
\end{aligned}
$$

We now test the robustness of our procedure with respect to underlying distributions of the error term by relaxing the normality assumption and using discrete distributions instead. 
Concretely, to set the distribution of the random variable $v_{i t}$, we first generate its support, $S_{v}$, by taking $N_{v}$ points from a uniform sampling over the interval [-1,1]. Then we draw $N_{v}$ i.i.d. random variables $\omega_{k} \sim$ Exponential(1). The probability mass assigned to each

point $s_{k} \in S_{v}$ is obtained by setting $p_{k}=\frac{\omega_{k}}{\sum_{i} \omega_{i}}$. Finally, we translate each point in $S_{v}$ so that $v_{i t}$ has zero mean. It is well known that the probability distribution obtained in this fashion is equivalent to a uniform sampling from a simplex in $N_{v}$ dimensional space. The construction of the simulated model follows exactly the case of the Normal distribution, with the only difference being the use of the discrete distribution described above in every place where the Normal distribution is used for $v_{i t}$.

\section{B. Simulation Results}

This section reports Monte Carlo simulations of our LIBMA methodology in order to assess its performance. We generate 100 instances of the data generating process with the exogenous variables $\mathbf{x}_{\mathbf{i t}}$, endogenous variables $\mathbf{w}_{\mathbf{i t}}$, and parameter values $\left(\begin{array}{ccc}\alpha & \theta_{x} & \theta_{w}\end{array}\right)^{\prime}$ as discussed in the previous section, and we present results in the form of medians, means, variances and quartiles. We consider several sample sizes, $N=200,500,1000$ and 2000, and several values for the coefficient of the lagged dependent variable, $\alpha=0.95,0.50$, and 0.30 . In the first set of simulations we assume that both the random error term $v_{i t}$ and the individual effect $\eta_{i}$ are drawn from a Normal distribution, $v_{i t} \sim N\left(0, \sigma_{v}^{2}\right)$ and $\eta_{i} \sim N\left(0, \sigma_{\eta}^{2}\right)$, respectively. We consider the cases where $\sigma_{v}=0.05,0.10$, and 0.50 while $\sigma_{\eta}=0.10$. Since our methodology should not depend on the normality of the random error term, we check for robustness by creating a second set of simulations where the assumption of normality for $v_{i t}$ is dropped, as discussed earlier.

\section{Model selection}

In the Bayesian framework, the posterior model probability is a key indicator of performance. Table 1 presents means, variances, and three quartiles (Q1, median, and Q3) for the posterior probability of the true model across the 100 instances. As expected, the mean posterior probabilities of the true model increase with the sample size. For sample size values of $N=200,500,1000$ and 2000, average values of the posterior model probability 
are about $0.31,0.46,0.57$, and 0.65 , respectively. Median posterior model probabilities are slightly higher than the means, with average values of $0.32,0.50,0.62$, and 0.69 . In addition, as the sample increases, the distribution becomes skewed toward 1. Quartiles and distribution plots show that as the sample increases the distributions of the posterior model probabilities are becoming less and less normal, with long left tails. ${ }^{13}$

As shown in (3) the posterior model probability depends on the prior model probability. Under the assumption that all models have equal prior probability, the more variables are under consideration the smaller the prior probability for each model. Obviously that has an effect on the absolute value of the posterior model probability. Therefore, we choose to also compute a relative measure that helps one understand how well the methodology performs. Table 2 presents the ratio of the posterior model probability of the true model to the highest posterior probability of all the other models (excluding the true model). On average this ratio is above unity for all the cases considered, suggesting that the correct model is on average favored over all the other models. As expected, the average ratios increase with the sample size, starting from about 2.26 for $N=200$ and reaching 7.09 for $N=2000$.

In Table 3 we examine how often our methodology recovers the true model by reporting how many times, out of 100 instances, the true model has the highest posterior probability. The results indicate that this is done quite well. For the smallest sample size, $N=200$, the recovery rate varies from 65 percent to 83 percent. For $N=500$ we see an improvement in the selection of the true model with the success rate ranging from 82 percent to 93 percent. For sample sizes bigger than 1000, the recovery rate stays over 90 percent, reaching 97 percent in a couple of cases.

\section{Model averaging}

While model selection properties are desired, researchers are often more interested in making inferences. Table 4 presents the posterior inclusion probabilities for all the variables considered along with the true model (column 2 of the table).${ }^{14}$ Given the assumptions made relative to the model priors, the prior probability of inclusion for each variable is the same and equal to 0.5. From Table 4 we see that the median value of the inclusion probability for all the relevant explanatory variables is greater than 0.942 in all cases considered. As the

\footnotetext{
${ }_{14}^{13}$ Figures 1, 2, 4, and 5 in Appendix A show density plots for the posteriors in Tables 1, 2, 6, and 7. ${ }^{14} \mathrm{~A}$ value of $1(0)$ in column 2 indicates that the true model contains (excludes) that variable.
} 
sample size increases the posterior inclusion probabilities approach 1 for all the relevant variables. In fact for sample sizes greater than 500, the median value of the probability of inclusion for all relevant variables is practically 1 . For the variables not contained in the true model the median posterior probability of inclusion decreases with the sample size with the upper bound being less than 0.076 for the case when $N=2000$.

We turn now to the parameter estimates, and examine how the estimated values compare with the true parameter values. Table 5 presents the median values of the estimated parameters, averaged over 100 replications, compared to the parameters of the true model. ${ }^{15}$ As in the case of inclusion probabilities, our methodology is performing very well in estimating the parameters, with the performance improving as the sample gets larger. In Figure 3 of Appendix A, we present the box plots for the parameter estimates of Table 5, for the case of $\alpha=0.95$ and $\sigma_{v}=0.1$. It becomes clear that as the sample increases the variance of the distribution decreases and the median converges to the true value. Aside from the fact that the estimates are very close to the true parameter values, the variance over the 100 replications is also very small across the board with values less than $10^{-5}$ in many cases.

\section{Robustness checks using non-Gaussian errors}

As discussed in Section A we perform robustness analysis by relaxing the normality assumption for the error term $v_{i t}$. Overall, as shown in Tables 6-10, the results are very similar to those presented in Tables 1-5. Tables 6 and 7 (which are analogous to Tables 1 and 2), present posterior model probabilities for the true model, and the ratio of the posterior model probability of the true model to the highest posterior probability of all other models, respectively. In Table 6, average mean and median values of the posterior model probability are about the same as in Table 1 . Median posterior model probabilities are again slightly higher than the means, a result that is consistent with a skewed distribution. In addition, as the sample increases, the posterior model probabilities increase and the distribution becomes increasingly more skewed toward 1. For Table 7, conclusions are similar to Table 2. On average, the ratio of the posterior model probability of the true model to the highest posterior probability of all other models is above unity for all cases considered

\footnotetext{
${ }^{15}$ Parameter values are discussed in section 4. Essentially these are constant for $x_{1}, x_{2}, x_{3}, x_{4}, x_{5}, x_{6}, w_{1}$ and $w_{2}$, and vary for $y_{t-1}$ based on the values of $\alpha=0.95,0.50$ and 0.30 .
} 
suggesting that the correct model is, on average, favored over all the other models. As expected, the average ratios increase with the sample size, starting from about 1.66 for $N=200$, and reaching 7.29 for $N=2000$.

Model recovery under non-Gaussian errors is still very good. As shown in Table 8, results are very similar to those of Table 3 . For the smallest sample size, $N=200$, the recovery rate varies from 65 percent to 82 percent. For $N=500$ we see an improvement in the selection of the true model with the success rate ranging from 81 percent to 91 percent. For the biggest sample size considered, $N=2000$, the recovery rate stays over 88 percent, reaching again 97 percent for $\alpha=0.30$.

Tables 9 and 10 present the posterior inclusion probabilities and parameter estimates using LIBMA and compares them the true model. From Table 9, we see that, the median inclusion probability for all the relevant explanatory variables is greater than 0.939 in all cases considered. As the sample size increases, the posterior inclusion probabilities approach 1 for all the relevant variables. As in the Gaussian case, for sample sizes greater than 500, the probability of inclusion for all relevant variables is practically 1 . For the variables not

contained in the true model the median posterior probability of inclusion decreases with the sample size with the upper bound being less than 0.078 for the case when $N=2000$. In Table 10, estimated parameter medians and variances are almost identical to those reported in Table 5. As in the Gaussian case, our methodology performs well by generating estimates close to the true parameter values for all types of variables, with performance improving with larger samples. In Figure 6 of Appendix A the box plots for the parameter estimates of Table 10 (for the case of $\alpha=0.95$ and $\sigma_{v}=0.1$ ) indicate that the variance of the distribution decreases and the median converges to the true value asymptotically.

\section{CONCLUSION}

This paper proposes a limited information methodology in the context of Bayesian Model Averaging, which we label LIBMA, for panel data models where the lagged dependent variable appears as a regressor and endogenous variables are present. The LIBMA methodology incorporates a GMM estimator for dynamic panel data models in a Bayesian Model Averaging framework to explicitly account for model uncertainty. Our methodology adds value to the existing literature in three important ways. First, while standard BMA is a full information technique where a complete stochastic specification is assumed, LIBMA is a limited information approach which relies on GMM estimation, a limited information 
technique based on moment restrictions rather than a complete stochastic specification. Second, LIBMA explicitly controls for endogeneity. The likelihood and exact expressions of the marginal likelihood used in the fully Bayesian analyses are replaced by the limited information construct modeled on the GMM estimation, and a limited information criterion as an approximation to the actual marginal likelihoods, respectively. Third, we use this methodology in a panel setting thus expanding its usability to a wide range of applications.

Based on simulation results, we conclude that asymptotically LIBMA performs very well and it can be used to address the issue of model uncertainty in the dynamic panel data models with endogenous regressors.

Future research should explore the possibility of using the LIBMA methodology for applications where the sample size is constrained by data availability, such as those investigating robust patterns of cross-country growth behavior. 


\section{REFERENCES}

Andrews, D. W. K., and B. Lu, 2001, "Consistent Model and Moment Selection Procedures for GMM Estimation with Application to Dynamic Panel Data Models," Journal of Econometrics, Vol. 101, No. 1, pp. 123-64.

Aragones, E., I. Gilboa, A. Postlewaite, and D. Schmeidler, 2005, "Fact-Free Learning," American Economic Review Vol. 95, No. 5, pp. 1355-68.

Back, K. and D. P. Brown, 1993, "Implied Probabilities in GMM Estimators", Econometrica, Vol. 61, No. 4, pp. 971-975.

Blundell, R., and S. Bond, 1998, "Initial Conditions and Moment Restrictions in Dynamic Panel Data Models," Journal of Econometrics, Vol. 87, No. 1, pp. 114-43.

Brock, W., and S. Durlauf, 2001, "Growth Empirics and Reality," World Bank Economic Review, 15, pp. 229-272.

Chernozhukov, V., and H. Hong, 2003, "An MCMC Approach to Classical Estimation," Journal of Econometrics, Vol. 115, No. 2, pp. 293-346.

Chipman, H., E.I. George, and R.E. McCulloch, 2001, "The Practical Implementation of Bayesian Model Selection," (with discussion) in P. Lahiri (ed), Model Selection IMS Lecture Notes, Vol. 38, pp. 70-134.

Durlauf, S., and D. Quah, 1999, "The New Empirics of Economic Growth," in J. B. Taylor and M. Woodford (eds), Handbook of Macroeconomics Vol. IA (North Holland).

Fernàndez C., E. Ley, and M. Steel, 2001a, "Model Uncertainty in Cross-Country Growth Regressions," Journal of Applied Econometrics, Vol. 16, pp. 563-76.

Fernàndez C., E. Ley and M.F.J. Steel, 2001b, "Benchmark Priors for Bayesian Model Averaging," Journal of Econometrics, Vol. 100, pp. 381-427.

Hall, A.R., 2005, Generalized Method of Moments (New York: Oxford University Press).

Hoeting, J.A., D. Madigan, A.E. Raftery, and C.T. Volinsky, 1999, "Bayesian Model Averaging: A Tutorial," Statistical Science, Vol. 14, No. 4, pp. 382-417.

Hong, H., and B. Preston, 2008, "Bayesian Averaging, Prediction and Nonnested Model Selection," NBER Working Papers 14284, National Bureau of Economic Research, Inc.

Jacobson T., and S. Karlsson, 2004, "Finding Good Predictors for Inflation: A Bayesian Model Averaging Approach", Journal of Forecasting, Vol. 23, pp. 476-496.

Kass, R., and A. Raftery, 1995, "Bayes Factors," Journal of the American Statistical Association, Vol. 90, No. 430, pp. 773-95. 
Kass, R., and L. Wasserman, 1995, "A Reference Bayesian Test for Nested Hypotheses and Its Relationship to the Schwarz Criterion," Journal of the American Statistical Association, Vol. 90, No. 431, pp. 928-34.

Kim, J. Y., 2002, "Limited Information Likelihood and Bayesian Analysis," Journal of Econometrics, Vol. 107, No. 1-2, pp. 175-93.

Koop, G., and L. Tole, 2004, "Measuring the Health Effects of Air Pollution: to What Extent Can We Really Say that People are Dying from Bad Air?" Journal of Environmental Economics and Management 47, January, pp. 30-54.

Leamer, E., 1978, Specification Searches: Ad Hoc Inference with Non-experimental Data (New York: Wiley).

Leamer, E., 1983, "Let's Take the Con Out of Econometrics," American Economic Review, Vol. 73, pp. 31-43.

Ley E., and M. Steel, 2008, "On the Effect of Prior Assumptions in Bayesian Model Averaging with Applications to Growth Regression," Journal of Applied Econometrics, forthcoming.

Madigan, D.M. and Raftery, A.E., 1994, "Model Selection and Accounting for Model Uncertainty in Graphical Models using Occam's Window," Journal of the American Statistical Association, Vol. 89, pp. 1335-1346.

Mitchell, T.J., and J.J. Beauchamp, 1988, "Bayesian Variable Selection in Linear Regression," Journal of the American Statistical Association Vol. 83, pp. 1023-1032.

Moral-Benito, E., 2007, "Determinants of Economic Growth: A Bayesian Panel Data Approach," CEMFI Working Papers Paper 0719.

Morales, K.H., J.G. Ibrahim, C. Chen, and L.M. Ryan, 2006, "Bayesian Model Averaging With Applications to Benchmark Dose Estimation for Arsenic in Drinking Water," Journal of the American Statistical Association 101, Vol. 473, pp. 9-17.

Moulton, B.R., 1991, "A Bayesian Approach to Regression Selection and Estimation with Application to a Price Index for Radio Services," Journal of Econometrics, Vol. 49, pp. 169-93.

Raftery, A. E., 1995, "Bayesian Model Selection in Social Research," Sociological Methodology, Vol. 25, pp. 111-163.

Raftery, A. E., 1996, "Approximate Bayes Factors and Accounting for Model Uncertainty in Generalized Linear Models," Biometrika, Vol. 83, pp. 251-66.

Raftery, A.E., D. Madigan, and J. A. Hoeting, 1997, "Bayesian Model Averaging for Linear Regression Models," Journal of the American Statistical Association, 92, pp. 179-191.

Ragusa, G., 2008, "Bayesian Likelihoods for Moment Condition Models," mimeo. 
Sala-i-Martin, X., Doppelhofer, G., R., and I. Miller, 2004, "Determinants of Long-Term Growth: A Bayesian Averaging of Classical Estimates (BACE) Approach," American Economic Review, Vol. 94, No. 4, pp. 813-35.

Schennach, S.C., 2005, "Bayesian Exponentially Tilted Empirical Likelihood," Biometrika, Vol. 92, pp. 31-46.

Tsangarides, C., 2004, "A Bayesian Approach to Model Uncertainty," IMF Working Paper No. 04/68 (Washington: International Monetary Fund).

Wintle, B.A., M.A. McCarthy, C.T. Volinsky, and R.P. Kavanagh, 2003, "The Use of Bayesian Model Averaging to Better Represent Uncertainty in Ecological Models," Conservation Biology 17, December, pp. 1579-1590.

Yeung, K.Y., R.E. Bumgarner, and A. E. Raftery, 2005, "Bayesian Model Averaging: Development of an Improved Multi-Class, Gene Selection and Classification Tool for Micro array Data," Bioinformatics 21, Vol. 10, pp. 2394-2402. 
Table 1. Posterior Probability of the True Model Summary statistics using LIBMA estimation for various $n$, a, and $s_{v}$

\begin{tabular}{|c|c|c|c|c|c|c|c|c|c|}
\hline \multirow[b]{3}{*}{ Sample } & \multicolumn{3}{|c|}{0.95} & \multicolumn{3}{|c|}{0.50} & \multicolumn{3}{|c|}{0.30} \\
\hline & 0.05 & 0.10 & $\overline{0.50}$ & 0.05 & 0.10 & $\overline{0.50}$ & 0.05 & 0.10 & 0.50 \\
\hline & & & & & & & & & \\
\hline \multicolumn{10}{|l|}{$N=200$} \\
\hline Mean & 0.318 & 0.328 & 0.286 & 0.341 & 0.331 & 0.258 & 0.326 & 0.333 & 0.255 \\
\hline Variance & 0.017 & 0.013 & 0.013 & 0.015 & 0.018 & 0.018 & 0.018 & 0.014 & 0.014 \\
\hline Q1 & 0.231 & 0.255 & 0.202 & 0.276 & 0.248 & 0.156 & 0.255 & 0.255 & 0.167 \\
\hline Median & 0.339 & 0.327 & 0.294 & 0.361 & 0.355 & 0.268 & 0.337 & 0.357 & 0.258 \\
\hline Q3 & 0.425 & 0.430 & 0.386 & 0.426 & 0.440 & 0.363 & 0.439 & 0.425 & 0.324 \\
\hline \multicolumn{10}{|l|}{$N=500$} \\
\hline Mean & 0.487 & 0.465 & 0.440 & 0.470 & 0.459 & 0.450 & 0.468 & 0.458 & 0.474 \\
\hline Variance & 0.017 & 0.021 & 0.021 & 0.023 & 0.024 & 0.019 & 0.022 & 0.023 & 0.020 \\
\hline Q1 & 0.426 & 0.378 & 0.336 & 0.449 & 0.378 & 0.363 & 0.391 & 0.339 & 0.417 \\
\hline Median & 0.519 & 0.501 & 0.467 & 0.523 & 0.501 & 0.475 & 0.493 & 0.502 & 0.528 \\
\hline Q3 & 0.576 & 0.578 & 0.562 & 0.567 & 0.577 & 0.559 & 0.592 & 0.570 & 0.579 \\
\hline \multicolumn{10}{|l|}{$N=1000$} \\
\hline Mean & 0.567 & 0.552 & 0.561 & 0.561 & 0.570 & 0.562 & 0.588 & 0.577 & 0.586 \\
\hline Variance & 0.026 & 0.025 & 0.021 & 0.023 & 0.024 & 0.022 & 0.018 & 0.026 & 0.021 \\
\hline Q1 & 0.509 & 0.473 & 0.498 & 0.490 & 0.513 & 0.489 & 0.545 & 0.543 & 0.525 \\
\hline Median & 0.622 & 0.602 & 0.611 & 0.605 & 0.632 & 0.604 & 0.632 & 0.623 & 0.628 \\
\hline Q3 & 0.679 & 0.668 & 0.666 & 0.671 & 0.685 & 0.676 & 0.678 & 0.682 & 0.698 \\
\hline \multicolumn{10}{|l|}{$N=2000$} \\
\hline Mean & 0.646 & 0.650 & 0.667 & 0.638 & 0.636 & 0.645 & 0.633 & 0.636 & 0.670 \\
\hline Variance & 0.020 & 0.019 & 0.014 & 0.027 & 0.025 & 0.027 & 0.021 & 0.018 & 0.015 \\
\hline Q1 & 0.605 & 0.570 & 0.614 & 0.573 & 0.549 & 0.611 & 0.567 & 0.574 & 0.641 \\
\hline Median & 0.690 & 0.698 & 0.705 & 0.704 & 0.686 & 0.701 & 0.680 & 0.655 & 0.700 \\
\hline Q3 & 0.748 & 0.747 & 0.750 & 0.740 & 0.748 & 0.752 & 0.738 & 0.736 & 0.757 \\
\hline
\end{tabular}

Notes:

1. For the idiosyncratic error term, $\eta_{i} \sim \mathrm{N}\left(0, \sigma_{\eta}{ }^{2}\right)$ where $\sigma_{\eta}=0.10$.

2. The error term is normally distributed $v_{i t} \sim \mathrm{N}\left(0, \sigma_{v}{ }^{2}\right)$. 
Table 2. Posterior Probability Ratio of True Model/Best Among the Other Models Summary statistics using LIBMA estimation for various $n, \alpha$, and $\sigma_{v}$

\begin{tabular}{|c|c|c|c|c|c|c|c|c|c|}
\hline \multirow[b]{3}{*}{ Sample } & \multicolumn{3}{|c|}{0.95} & \multicolumn{3}{|c|}{0.50} & \multicolumn{3}{|c|}{0.30} \\
\hline & 0.05 & 0.10 & 0.50 & 0.05 & 0.10 & 0.50 & 0.05 & 0.10 & 0.50 \\
\hline & & & & & & & & & \\
\hline \multicolumn{10}{|l|}{$N=200$} \\
\hline Mean & 2.044 & 2.102 & 2.061 & 2.255 & 2.201 & 1.809 & 2.117 & 2.172 & 1.681 \\
\hline Variance & 1.932 & 1.627 & 1.655 & 1.773 & 2.089 & 1.709 & 1.928 & 1.740 & 1.332 \\
\hline Q1 & 0.902 & 1.118 & 0.921 & 1.339 & 0.980 & 0.689 & 1.007 & 1.027 & 0.710 \\
\hline Median & 1.715 & 1.810 & 1.848 & 2.031 & 1.991 & 1.611 & 1.927 & 2.010 & 1.546 \\
\hline Q3 & 3.197 & 3.015 & 2.968 & 3.046 & 3.276 & 2.609 & 3.317 & 3.021 & 2.483 \\
\hline \multicolumn{10}{|l|}{$N=500$} \\
\hline Mean & 3.729 & 3.486 & 3.151 & 3.456 & 3.372 & 3.094 & 3.583 & 3.399 & 3.594 \\
\hline Variance & 4.285 & 4.292 & 4.405 & 4.083 & 4.823 & 3.583 & 5.232 & 4.795 & 4.126 \\
\hline Q1 & 2.145 & 1.515 & 1.568 & 2.066 & 1.535 & 1.646 & 1.607 & 1.623 & 1.950 \\
\hline Median & 3.538 & 3.479 & 2.692 & 3.377 & 3.282 & 2.921 & 3.078 & 3.186 & 3.537 \\
\hline Q3 & 5.063 & 4.998 & 4.409 & 4.815 & 4.901 & 4.476 & 5.496 & 4.644 & 5.185 \\
\hline \multicolumn{10}{|l|}{$N=1000$} \\
\hline Mean & 5.174 & 4.797 & 4.732 & 4.809 & 5.155 & 4.911 & 5.420 & 5.208 & 5.621 \\
\hline Variance & 10.470 & 9.862 & 7.615 & 9.301 & 9.078 & 9.004 & 9.109 & 8.798 & 11.017 \\
\hline Q1 & 2.811 & 2.098 & 2.380 & 2.146 & 2.318 & 2.163 & 3.189 & 2.802 & 2.819 \\
\hline Median & 4.754 & 4.363 & 4.682 & 4.530 & 5.080 & 4.924 & 5.211 & 4.895 & 5.743 \\
\hline Q3 & 7.167 & 7.065 & 6.629 & 6.744 & 7.530 & 7.233 & 7.588 & 7.644 & 8.412 \\
\hline \multicolumn{10}{|l|}{$N=2000$} \\
\hline Mean & 6.479 & 6.808 & 7.088 & 6.777 & 6.592 & 6.971 & 6.240 & 6.200 & 6.881 \\
\hline Variance & 14.244 & 19.297 & 15.844 & 18.505 & 18.891 & 18.147 & 15.645 & 17.566 & 15.194 \\
\hline Q1 & 3.144 & 3.526 & 3.811 & 3.035 & 2.826 & 3.642 & 3.159 & 2.873 & 4.086 \\
\hline Median & 6.616 & 6.500 & 6.892 & 6.597 & 5.822 & 6.714 & 5.359 & 5.223 & 6.078 \\
\hline Q3 & 9.657 & 8.884 & 10.054 & 9.258 & 9.360 & 10.253 & 9.033 & 8.732 & 9.433 \\
\hline
\end{tabular}

Notes:

1. For the idiosyncratic error term, $\eta_{i} \sim \mathrm{N}\left(0, \sigma_{\eta}{ }^{2}\right)$ where $\sigma_{\eta}=0.10$.

2. The error term is normally distributed $v_{i t} \sim \mathrm{N}\left(0, \sigma_{v}{ }^{2}\right)$. 
Table 3. Probability of Retrieving the True Model Summary statistics using LIBMA estimation for various $n, \alpha$, and $\sigma_{v}$

\begin{tabular}{|c|c|c|c|c|c|c|c|c|c|}
\hline \multirow[b]{3}{*}{ Sample } & \multicolumn{3}{|c|}{0.95} & \multicolumn{3}{|c|}{0.50} & \multicolumn{3}{|c|}{0.30} \\
\hline & 0.05 & 0.10 & 0.50 & 0.05 & 0.10 & 0.50 & 0.05 & 0.10 & 0.50 \\
\hline & & & & & & & & & \\
\hline \multicolumn{10}{|l|}{$N=200$} \\
\hline \% Correct & 74 & 78 & 74 & 83 & 73 & 65 & 76 & 76 & 65 \\
\hline \multicolumn{10}{|l|}{$N=500$} \\
\hline$\%$ Correct & 91 & 87 & 83 & 83 & 84 & 83 & 85 & 82 & 87 \\
\hline \multicolumn{10}{|l|}{$N=1000$} \\
\hline$\%$ Correct & 91 & 92 & 90 & 90 & 91 & 91 & 91 & 92 & 90 \\
\hline \multicolumn{10}{|l|}{$N=2000$} \\
\hline \% Correct & 94 & 93 & 97 & 92 & 94 & 92 & 92 & 94 & 97 \\
\hline
\end{tabular}

Notes:

1. For the idiosyncratic error term, $\eta_{i} \sim \mathrm{N}\left(0, \sigma_{\eta}{ }^{2}\right)$ where $\sigma_{\eta}=0.10$.

2. The error term is normally distributed $v_{i t} \sim \mathrm{N}\left(0, \sigma_{v}{ }^{2}\right)$. 
31

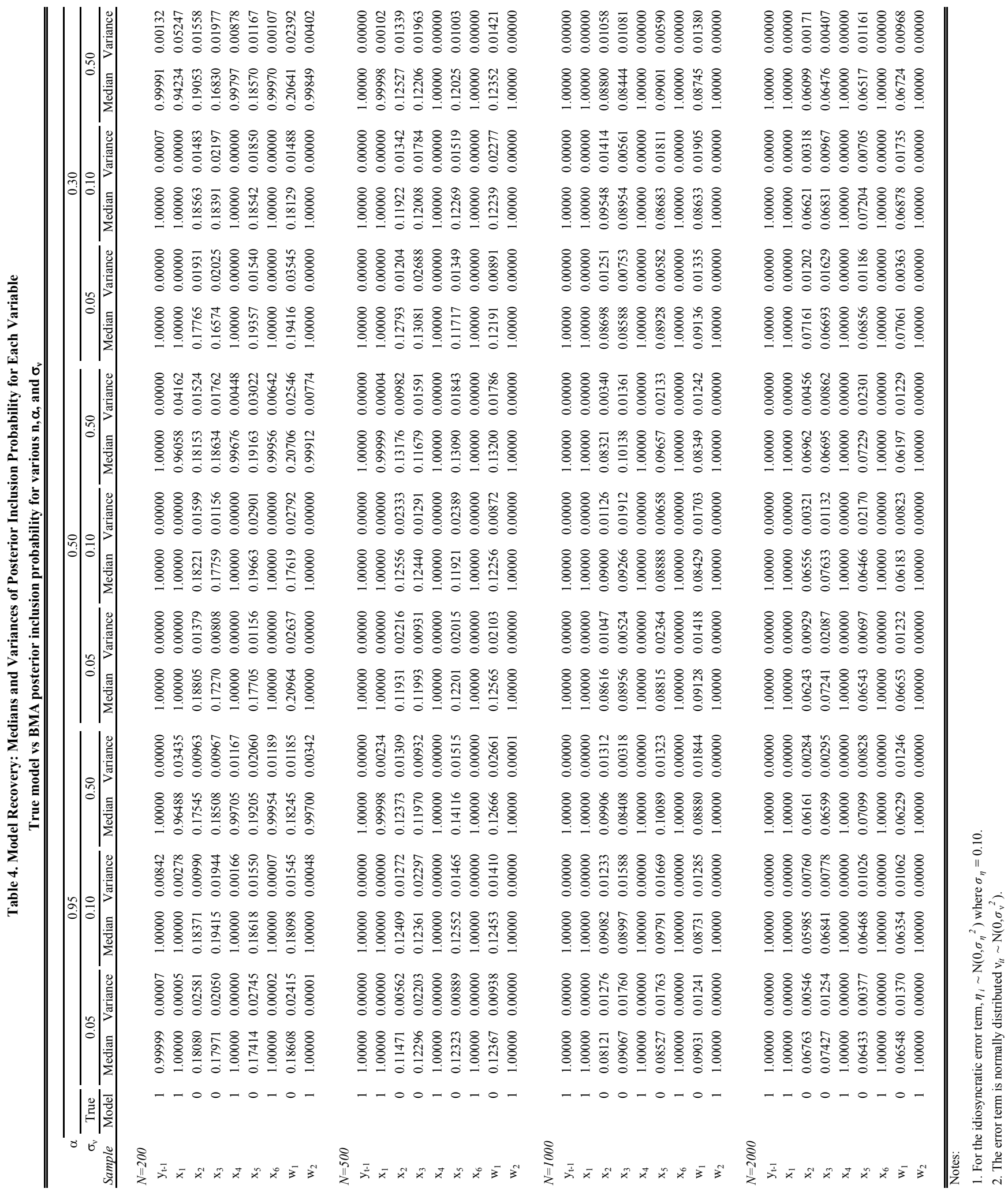


32

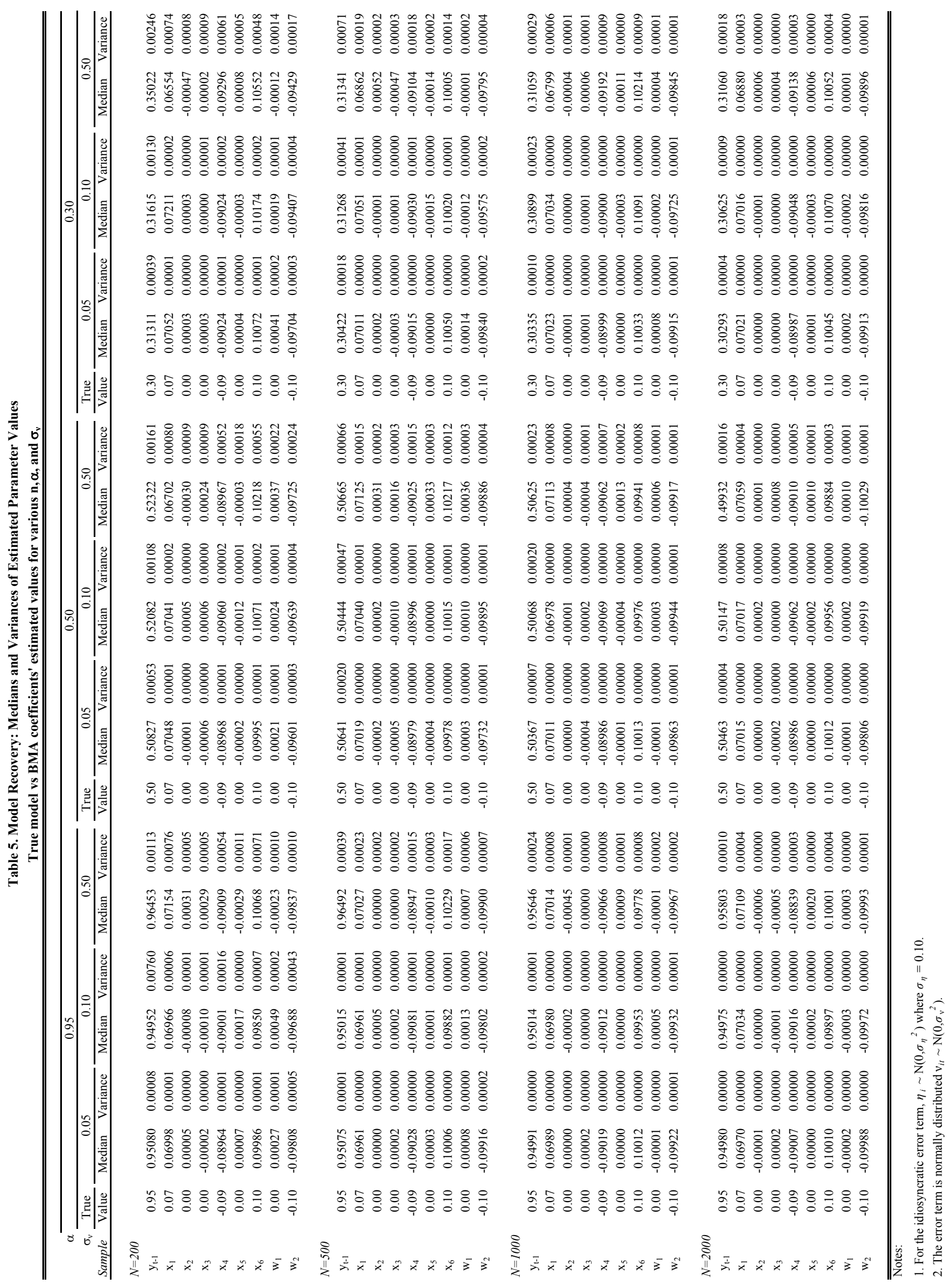


Table 6. Posterior Probability of the True Model Summary statistics using LIBMA estimation for various $n, \alpha$, and $\sigma_{v}$

\begin{tabular}{|c|c|c|c|c|c|c|c|c|c|}
\hline \multirow[b]{3}{*}{ Sample } & \multicolumn{3}{|c|}{0.95} & \multicolumn{3}{|c|}{0.50} & \multicolumn{3}{|c|}{0.30} \\
\hline & 0.05 & 0.10 & 0.50 & 0.05 & 0.10 & 0.50 & 0.05 & 0.10 & 0.50 \\
\hline & & & & & & & & & \\
\hline \multicolumn{10}{|l|}{$N=200$} \\
\hline Mean & 0.344 & 0.318 & 0.240 & 0.349 & 0.328 & 0.262 & 0.332 & 0.319 & 0.252 \\
\hline Variance & 0.017 & 0.015 & 0.017 & 0.014 & 0.021 & 0.017 & 0.017 & 0.018 & 0.019 \\
\hline Q1 & 0.273 & 0.223 & 0.127 & 0.284 & 0.231 & 0.145 & 0.229 & 0.212 & 0.146 \\
\hline Median & 0.379 & 0.321 & 0.244 & 0.370 & 0.355 & 0.278 & 0.360 & 0.349 & 0.253 \\
\hline Q3 & 0.454 & 0.414 & 0.342 & 0.449 & 0.453 & 0.356 & 0.435 & 0.433 & 0.366 \\
\hline \multicolumn{10}{|l|}{$N=500$} \\
\hline Mean & 0.476 & 0.470 & 0.437 & 0.474 & 0.460 & 0.479 & 0.478 & 0.501 & 0.470 \\
\hline Variance & 0.025 & 0.020 & 0.026 & 0.019 & 0.027 & 0.018 & 0.022 & 0.017 & 0.021 \\
\hline Q1 & 0.410 & 0.397 & 0.378 & 0.393 & 0.373 & 0.386 & 0.388 & 0.454 & 0.402 \\
\hline Median & 0.523 & 0.499 & 0.463 & 0.515 & 0.518 & 0.534 & 0.528 & 0.538 & 0.510 \\
\hline Q3 & 0.604 & 0.594 & 0.567 & 0.585 & 0.588 & 0.580 & 0.585 & 0.603 & 0.584 \\
\hline \multicolumn{10}{|l|}{$N=1000$} \\
\hline Mean & 0.532 & 0.538 & 0.549 & 0.549 & 0.564 & 0.553 & 0.564 & 0.578 & 0.567 \\
\hline Variance & 0.031 & 0.031 & 0.028 & 0.026 & 0.023 & 0.022 & 0.027 & 0.028 & 0.020 \\
\hline Q1 & 0.406 & 0.451 & 0.509 & 0.493 & 0.492 & 0.497 & 0.516 & 0.525 & 0.518 \\
\hline Median & 0.603 & 0.578 & 0.595 & 0.597 & 0.605 & 0.587 & 0.624 & 0.642 & 0.610 \\
\hline Q3 & 0.664 & 0.675 & 0.668 & 0.662 & 0.672 & 0.657 & 0.674 & 0.692 & 0.669 \\
\hline \multicolumn{10}{|l|}{$N=2000$} \\
\hline Mean & 0.598 & 0.633 & 0.606 & 0.626 & 0.657 & 0.667 & 0.651 & 0.668 & 0.660 \\
\hline Variance & 0.039 & 0.019 & 0.028 & 0.034 & 0.021 & 0.016 & 0.020 & 0.014 & 0.018 \\
\hline Q1 & 0.506 & 0.580 & 0.513 & 0.562 & 0.624 & 0.627 & 0.598 & 0.630 & 0.638 \\
\hline Median & 0.669 & 0.669 & 0.657 & 0.699 & 0.710 & 0.717 & 0.708 & 0.706 & 0.699 \\
\hline Q3 & 0.742 & 0.734 & 0.737 & 0.756 & 0.753 & 0.752 & 0.742 & 0.751 & 0.746 \\
\hline
\end{tabular}

Notes:

1. The error terms are constructed using discrete distributions (see section IV.A.). 
Table 7. Posterior Probability Ratio: True Model/Best Among the Other Models Summary statistics using LIBMA estimation for various $n, \alpha$, and $\sigma_{v}$

\begin{tabular}{|c|c|c|c|c|c|c|c|c|c|}
\hline \multirow{3}{*}{ Sample $^{\alpha}{ }^{\alpha}$} & \multicolumn{3}{|c|}{0.95} & \multicolumn{3}{|c|}{0.50} & \multicolumn{3}{|c|}{0.30} \\
\hline & 0.05 & 0.10 & 0.50 & 0.05 & 0.10 & 0.50 & 0.05 & 0.10 & 0.50 \\
\hline & & & & & & & & & \\
\hline \multicolumn{10}{|l|}{$N=200$} \\
\hline Mean & 2.282 & 2.040 & 1.659 & 2.323 & 2.242 & 1.798 & 2.175 & 2.080 & 1.820 \\
\hline Variance & 1.981 & 2.000 & 1.606 & 1.726 & 2.321 & 1.629 & 1.803 & 1.932 & 1.822 \\
\hline Q1 & 1.230 & 0.867 & 0.690 & 1.304 & 0.949 & 0.730 & 1.047 & 0.873 & 0.707 \\
\hline Median & 2.111 & 1.823 & 1.353 & 2.196 & 1.908 & 1.522 & 2.152 & 2.047 & 1.461 \\
\hline Q3 & 3.502 & 3.050 & 2.417 & 3.348 & 3.466 & 2.475 & 3.213 & 3.064 & 2.690 \\
\hline \multicolumn{10}{|l|}{$N=500$} \\
\hline Mean & 3.738 & 3.567 & 3.175 & 3.563 & 3.596 & 3.681 & 3.704 & 3.882 & 3.504 \\
\hline Variance & 5.286 & 4.471 & 4.485 & 4.499 & 5.716 & 4.507 & 4.530 & 4.605 & 4.756 \\
\hline Q1 & 1.784 & 1.809 & 1.526 & 2.031 & 1.564 & 1.797 & 1.651 & 2.021 & 1.572 \\
\hline Median & 3.809 & 3.196 & 2.959 & 3.128 & 3.430 & 3.899 & 3.860 & 3.682 & 3.570 \\
\hline Q3 & 5.677 & 5.719 & 4.581 & 5.223 & 5.279 & 5.189 & 5.422 & 5.816 & 5.368 \\
\hline \multicolumn{10}{|l|}{$N=1000$} \\
\hline Mean & 4.482 & 4.651 & 4.645 & 4.578 & 5.034 & 4.693 & 4.995 & 5.686 & 4.969 \\
\hline Variance & 9.738 & 10.456 & 8.166 & 8.924 & 9.303 & 9.957 & 8.401 & 11.604 & 8.523 \\
\hline Q1 & 1.634 & 1.813 & 2.436 & 2.232 & 2.226 & 2.468 & 2.657 & 2.674 & 2.826 \\
\hline Median & 4.213 & 3.746 & 4.760 & 3.775 & 4.945 & 4.029 & 5.106 & 5.878 & 4.703 \\
\hline Q3 & 6.376 & 7.137 & 6.650 & 6.789 & 7.405 & 6.868 & 7.220 & 8.211 & 7.094 \\
\hline \multicolumn{10}{|l|}{$N=2000$} \\
\hline Mean & 6.061 & 5.997 & 5.740 & 6.569 & 7.008 & 7.180 & 6.699 & 7.293 & 6.669 \\
\hline Variance & 18.867 & 14.406 & 16.746 & 19.530 & 15.723 & 15.063 & 14.792 & 16.717 & 13.738 \\
\hline Q1 & 2.663 & 2.953 & 1.912 & 3.268 & 4.008 & 4.345 & 3.288 & 3.937 & 3.740 \\
\hline Median & 5.228 & 5.302 & 5.160 & 6.278 & 6.977 & 7.028 & 6.701 & 6.917 & 6.427 \\
\hline Q3 & 9.679 & 8.340 & 8.646 & 9.696 & 9.771 & 10.033 & 9.571 & 10.203 & 9.015 \\
\hline
\end{tabular}

Notes:

1. The error terms are constructed using discrete distributions (see section IV.A.). 
Table 8. Probability of Retrieving the True Model Summary statistics using LIBMA estimation for various $n, \alpha$, and $\sigma_{v}$

\begin{tabular}{|c|c|c|c|c|c|c|c|c|c|}
\hline \multirow[b]{3}{*}{ Sample } & \multicolumn{3}{|c|}{0.95} & \multicolumn{3}{|c|}{0.50} & \multicolumn{3}{|c|}{0.30} \\
\hline & 0.05 & 0.10 & 0.50 & 0.05 & 0.10 & 0.50 & 0.05 & 0.10 & 0.50 \\
\hline & & & & & & & & & \\
\hline \multicolumn{10}{|l|}{$N=200$} \\
\hline$\%$ Correct & 78 & 70 & 65 & 82 & 72 & 71 & 76 & 70 & 66 \\
\hline \multicolumn{10}{|l|}{$N=500$} \\
\hline \% Correct & 87 & 87 & 81 & 90 & 84 & 88 & 85 & 91 & 83 \\
\hline \multicolumn{10}{|l|}{$N=1000$} \\
\hline$\%$ Correct & 82 & 88 & 89 & 88 & 91 & 87 & 87 & 90 & 92 \\
\hline \multicolumn{10}{|l|}{$N=2000$} \\
\hline \% Correct & 88 & 94 & 89 & 89 & 92 & 92 & 96 & 97 & 96 \\
\hline
\end{tabular}

Notes:

1. The error terms are constructed using discrete distributions (see section IV.A.). 
Table 9. Model Recovery: Medians and Variances of Posterior Inclusion Probability for Each Variable

True model vs BMA posterior inclusion probability for various $\mathbf{n}, \alpha$, and $\sigma_{v}$

\begin{tabular}{|c|c|c|c|c|c|c|c|c|c|c|c|c|c|c|c|c|c|c|c|}
\hline \multirow{3}{*}{$\begin{array}{r}\alpha \\
\sigma_{v} \\
\text { Sample } \\
\end{array}$} & \multirow{3}{*}{$\frac{}{\frac{\text { True }}{\text { Model }}}$} & \multicolumn{6}{|c|}{0.95} & \multicolumn{6}{|c|}{0.50} & \multicolumn{6}{|c|}{0.30} \\
\hline & & \multicolumn{2}{|c|}{0.05} & \multicolumn{2}{|c|}{0.10} & \multicolumn{2}{|c|}{0.50} & \multicolumn{2}{|c|}{0.05} & \multicolumn{2}{|c|}{0.10} & \multicolumn{2}{|c|}{0.50} & \multicolumn{2}{|c|}{0.05} & \multicolumn{2}{|c|}{0.10} & \multicolumn{2}{|c|}{0.50} \\
\hline & & Median & Variance & Median & Variance & Median & Variance & Median & Variance & Median & Variance & Median & Variance & Median & Variance & Median & Variance & Median & Variance \\
\hline \multicolumn{20}{|l|}{$N=200$} \\
\hline $\mathrm{y}_{\mathrm{t}-1}$ & 1 & 1.00000 & 0.00645 & 0.99999 & 0.00020 & 1.00000 & 0.00000 & 1.00000 & 0.00000 & 1.00000 & 0.00276 & 1.00000 & 0.00000 & 1.00000 & 0.00000 & 0.99999 & 0.00298 & 0.99974 & 0.00428 \\
\hline $\mathrm{x}_{1}$ & 1 & 1.00000 & 0.00464 & 0.99999 & 0.00003 & 0.93729 & 0.04238 & 1.00000 & 0.00000 & 1.00000 & 0.00000 & 0.95587 & 0.04862 & 1.00000 & 0.00000 & 1.00000 & 0.00000 & 0.93931 & 0.04225 \\
\hline $\mathrm{x}_{2}$ & 0 & 0.17454 & 0.02173 & 0.17474 & 0.01661 & 0.17118 & 0.01707 & 0.18556 & 0.00762 & 0.17447 & 0.02088 & 0.18673 & 0.02439 & 0.17292 & 0.01695 & 0.17668 & 0.01679 & 0.16842 & 0.01449 \\
\hline$x_{3}$ & 0 & 0.17667 & 0.02116 & 0.19481 & 0.01840 & 0.18169 & 0.02376 & 0.19783 & 0.01477 & 0.17500 & 0.02482 & 0.19002 & 0.01603 & 0.18012 & 0.01559 & 0.17240 & 0.01023 & 0.17422 & 0.02975 \\
\hline $\mathrm{x}_{4}$ & 1 & 1.00000 & 0.00001 & 1.00000 & 0.00005 & 0.99655 & 0.02350 & 1.00000 & 0.00000 & 1.00000 & 0.00000 & 0.99874 & 0.01165 & 1.00000 & 0.00000 & 1.00000 & 0.00000 & 0.99580 & 0.00891 \\
\hline $\mathrm{x}_{5}$ & 0 & 0.17948 & 0.01703 & 0.19036 & 0.02538 & 0.17095 & 0.00980 & 0.17557 & 0.01284 & 0.17196 & 0.01868 & 0.18045 & 0.00763 & 0.18280 & 0.02120 & 0.19580 & 0.03906 & 0.19070 & 0.01876 \\
\hline $\mathrm{x}_{6}$ & 1 & 1.00000 & 0.00264 & 1.00000 & 0.00001 & 0.99933 & 0.00683 & 1.00000 & 0.00000 & 1.00000 & 0.00000 & 0.99965 & 0.00122 & 1.00000 & 0.00000 & 1.00000 & 0.00000 & 0.99897 & 0.00719 \\
\hline $\mathrm{w}_{1}$ & 0 & 0.16520 & 0.01047 & 0.17782 & 0.02025 & 0.21088 & 0.03771 & 0.18629 & 0.02511 & 0.19334 & 0.03158 & 0.19282 & 0.03017 & 0.19512 & 0.02774 & 0.17923 & 0.02152 & 0.18846 & 0.03213 \\
\hline $\mathrm{w}_{2}$ & 1 & 1.00000 & 0.00004 & 0.99999 & 0.00008 & 0.99896 & 0.01245 & 1.00000 & 0.00000 & 1.00000 & 0.00000 & 0.99871 & 0.01455 & 1.00000 & 0.00000 & 1.00000 & 0.00002 & 0.99905 & 0.01706 \\
\hline \multicolumn{20}{|l|}{$N=500$} \\
\hline $\mathrm{y}_{\mathrm{t}-1}$ & 1 & 1.00000 & 0.00000 & 1.00000 & 0.00000 & 1.00000 & 0.00000 & 1.00000 & 0.00000 & 1.00000 & 0.00000 & 1.00000 & 0.00000 & 1.00000 & 0.00000 & 1.00000 & 0.00000 & 1.00000 & 0.00000 \\
\hline $\mathrm{x}_{1}$ & 1 & 1.00000 & 0.00000 & 1.00000 & 0.00000 & 0.99998 & 0.00070 & 1.00000 & 0.00000 & 1.00000 & 0.00000 & 0.99998 & 0.00009 & 1.00000 & 0.00000 & 1.00000 & 0.00000 & 1.00000 & 0.00000 \\
\hline $\mathrm{x}_{2}$ & 0 & 0.12089 & 0.01624 & 0.12964 & 0.01162 & 0.12260 & 0.01910 & 0.12530 & 0.01152 & 0.12395 & 0.01814 & 0.11977 & 0.01275 & 0.12902 & 0.01562 & 0.11685 & 0.01298 & 0.11901 & 0.01519 \\
\hline $\mathrm{x}_{3}$ & 0 & 0.11402 & 0.00929 & 0.13206 & 0.01191 & 0.13164 & 0.01376 & 0.12678 & 0.01312 & 0.11890 & 0.02294 & 0.12262 & 0.01901 & 0.11613 & 0.00696 & 0.11594 & 0.00213 & 0.12111 & 0.02191 \\
\hline $\mathrm{x}_{4}$ & 1 & 1.00000 & 0.00000 & 1.00000 & 0.00000 & 1.00000 & 0.00000 & 1.00000 & 0.00000 & 1.00000 & 0.00000 & 1.00000 & 0.00000 & 1.00000 & 0.00000 & 1.00000 & 0.00000 & 1.00000 & 0.00000 \\
\hline $\mathrm{x}_{5}$ & 0 & 0.11929 & 0.02669 & 0.11284 & 0.02250 & 0.12951 & 0.03295 & 0.12776 & 0.02185 & 0.12074 & 0.01966 & 0.13283 & 0.01079 & 0.12515 & 0.01542 & 0.11329 & 0.01032 & 0.11822 & 0.01197 \\
\hline $\mathrm{x}_{6}$ & 1 & 1.00000 & 0.00000 & 1.00000 & 0.00000 & 1.00000 & 0.00000 & 1.00000 & 0.00000 & 1.00000 & 0.00000 & 1.00000 & 0.00000 & 1.00000 & 0.00000 & 1.00000 & 0.00000 & 1.00000 & 0.00000 \\
\hline $\mathrm{w}_{1}$ & 0 & 0.11260 & 0.01555 & 0.12982 & 0.01207 & 0.13208 & 0.02213 & 0.12110 & 0.00725 & 0.12079 & 0.01688 & 0.11993 & 0.01018 & 0.11700 & 0.02356 & 0.12405 & 0.01790 & 0.12476 & 0.01897 \\
\hline $\mathrm{w}_{2}$ & 1 & 1.00000 & 0.00000 & 1.00000 & 0.00000 & 1.00000 & 0.00000 & 1.00000 & 0.00000 & 1.00000 & 0.00000 & 1.00000 & 0.00000 & 1.00000 & 0.00000 & 1.00000 & 0.00000 & 1.00000 & 0.00000 \\
\hline \multicolumn{20}{|l|}{$N=1000$} \\
\hline $\mathrm{y}_{\mathrm{t}-1}$ & 1 & 1.00000 & 0.00000 & 1.00000 & 0.00000 & 1.00000 & 0.00000 & 1.00000 & 0.00000 & 1.00000 & 0.00000 & 1.00000 & 0.00000 & 1.00000 & 0.00000 & 1.00000 & 0.00000 & 1.00000 & 0.00000 \\
\hline $\mathrm{x}_{1}$ & 1 & 1.00000 & 0.00000 & 1.00000 & 0.00000 & 1.00000 & 0.00000 & 1.00000 & 0.00000 & 1.00000 & 0.00000 & 1.00000 & 0.00000 & 1.00000 & 0.00000 & 1.00000 & 0.00000 & 1.00000 & 0.00000 \\
\hline $\mathrm{x}_{2}$ & 0 & 0.08629 & 0.01338 & 0.08669 & 0.01975 & 0.09677 & 0.01757 & 0.09342 & 0.01974 & 0.09442 & 0.00916 & 0.09734 & 0.00960 & 0.08971 & 0.00402 & 0.08278 & 0.00666 & 0.09309 & 0.01893 \\
\hline $\mathrm{x}_{3}$ & 0 & 0.09391 & 0.01813 & 0.08700 & 0.01796 & 0.09776 & 0.01077 & 0.09500 & 0.02258 & 0.10320 & 0.02065 & 0.08946 & 0.01944 & 0.08979 & 0.01938 & 0.08941 & 0.01775 & 0.09020 & 0.01212 \\
\hline $\mathrm{x}_{4}$ & 1 & 1.00000 & 0.00000 & 1.00000 & 0.00000 & 1.00000 & 0.00000 & 1.00000 & 0.00000 & 1.00000 & 0.00000 & 1.00000 & 0.00000 & 1.00000 & 0.00000 & 1.00000 & 0.00000 & 1.00000 & 0.00000 \\
\hline $\mathrm{x}_{5}$ & 0 & 0.09265 & 0.02334 & 0.09357 & 0.01437 & 0.08951 & 0.01987 & 0.08936 & 0.01272 & 0.08673 & 0.00974 & 0.09722 & 0.01832 & 0.09423 & 0.01871 & 0.08965 & 0.02386 & 0.09219 & 0.00721 \\
\hline $\mathrm{x}_{6}$ & 1 & 1.00000 & 0.00000 & 1.00000 & 0.00000 & 1.00000 & 0.00000 & 1.00000 & 0.00000 & 1.00000 & 0.00000 & 1.00000 & 0.00000 & 1.00000 & 0.00000 & 1.00000 & 0.00000 & 1.00000 & 0.00000 \\
\hline $\mathrm{w}_{1}$ & 0 & 0.08340 & 0.02110 & 0.09592 & 0.01865 & 0.10312 & 0.01823 & 0.08615 & 0.00752 & 0.08598 & 0.00875 & 0.09037 & 0.00482 & 0.08845 & 0.01810 & 0.08504 & 0.01168 & 0.08700 & 0.00789 \\
\hline $\mathrm{w}_{2}$ & 1 & 1.00000 & 0.00000 & 1.00000 & 0.00000 & 1.00000 & 0.00000 & 1.00000 & 0.00000 & 1.00000 & 0.00000 & 1.00000 & 0.00000 & 1.00000 & 0.00000 & 1.00000 & 0.00000 & 1.00000 & 0.00000 \\
\hline \multicolumn{20}{|l|}{$N=2000$} \\
\hline $\mathrm{y}_{\mathrm{t}-1}$ & 1 & 1.00000 & 0.00000 & 1.00000 & 0.00000 & 1.00000 & 0.00000 & 1.00000 & 0.00000 & 1.00000 & 0.00000 & 1.00000 & 0.00000 & 1.00000 & 0.00000 & 1.00000 & 0.00000 & 1.00000 & 0.00000 \\
\hline $\mathrm{x}_{1}$ & 1 & 1.00000 & 0.00000 & 1.00000 & 0.00000 & 1.00000 & 0.00000 & 1.00000 & 0.00000 & 1.00000 & 0.00000 & 1.00000 & 0.00000 & 1.00000 & 0.00000 & 1.00000 & 0.00000 & 1.00000 & 0.00000 \\
\hline $\mathrm{x}_{2}$ & 0 & 0.06555 & 0.01849 & 0.06911 & 0.00717 & 0.07534 & 0.01617 & 0.06574 & 0.01595 & 0.06419 & 0.01371 & 0.07118 & 0.00780 & 0.06999 & 0.01023 & 0.06780 & 0.00451 & 0.06292 & 0.00453 \\
\hline$x_{3}$ & 0 & 0.07132 & 0.01704 & 0.07693 & 0.00665 & 0.06512 & 0.00897 & 0.06166 & 0.01239 & 0.07266 & 0.00357 & 0.06714 & 0.00657 & 0.06812 & 0.00899 & 0.06538 & 0.00607 & 0.07263 & 0.02124 \\
\hline $\mathrm{x}_{4}$ & 1 & 1.00000 & 0.00000 & 1.00000 & 0.00000 & 1.00000 & 0.00000 & 1.00000 & 0.00000 & 1.00000 & 0.00000 & 1.00000 & 0.00000 & 1.00000 & 0.00000 & 1.00000 & 0.00000 & 1.00000 & 0.00000 \\
\hline $\mathrm{x}_{5}$ & 0 & 0.07611 & 0.02266 & 0.06596 & 0.00551 & 0.06860 & 0.01023 & 0.07207 & 0.01844 & 0.06466 & 0.01515 & 0.06562 & 0.00570 & 0.06948 & 0.00422 & 0.06723 & 0.00586 & 0.06441 & 0.00261 \\
\hline $\mathrm{x}_{6}$ & 1 & 1.00000 & 0.00000 & 1.00000 & 0.00000 & 1.00000 & 0.00000 & 1.00000 & 0.00000 & 1.00000 & 0.00000 & 1.00000 & 0.00000 & 1.00000 & 0.00000 & 1.00000 & 0.00000 & 1.00000 & 0.00000 \\
\hline $\mathrm{w}_{1}$ & 0 & 0.06935 & 0.02144 & 0.07806 & 0.01694 & 0.07013 & 0.02338 & 0.06401 & 0.01526 & 0.06688 & 0.00465 & 0.06322 & 0.00984 & 0.06317 & 0.01193 & 0.06128 & 0.00944 & 0.06257 & 0.00458 \\
\hline $\mathrm{w}_{2}$ & 1 & 1.00000 & 0.00000 & 1.00000 & 0.00000 & 1.00000 & 0.00000 & 1.00000 & 0.00000 & 1.00000 & 0.00000 & 1.00000 & 0.00000 & 1.00000 & 0.00000 & 1.00000 & 0.00000 & 1.00000 & 0.00000 \\
\hline
\end{tabular}

1. The error terms are constructed using discrete distributions (see section IV.A.). 
Table 10. Model Recovery: Medians and Variances of Estimated Parameter Values

True model vs BMA coefficients' estimated values for various $\mathbf{n}, \alpha$, and $\sigma_{y}$

\begin{tabular}{|c|c|c|c|c|c|c|c|c|c|c|c|c|c|c|c|c|c|c|c|c|c|}
\hline \multirow{3}{*}{$\begin{array}{r}\alpha \\
\sigma_{v} \\
\text { Sample }\end{array}$} & \multicolumn{7}{|c|}{0.95} & \multicolumn{7}{|c|}{0.50} & \multicolumn{7}{|c|}{0.30} \\
\hline & \multirow{2}{*}{$\begin{array}{l}\text { True } \\
\text { Value } \\
\end{array}$} & \multicolumn{2}{|c|}{0.05000} & \multicolumn{2}{|c|}{0.10000} & \multicolumn{2}{|c|}{0.50000} & \multirow{2}{*}{$\begin{array}{c}\text { True } \\
\text { Value } \\
\end{array}$} & \multicolumn{2}{|c|}{0.05000} & \multicolumn{2}{|c|}{0.10000} & \multicolumn{2}{|c|}{0.50000} & \multirow{2}{*}{$\begin{array}{l}\text { True } \\
\text { Value } \\
\end{array}$} & \multicolumn{2}{|c|}{0.05000} & \multicolumn{2}{|c|}{0.10000} & \multicolumn{2}{|c|}{0.50000} \\
\hline & & Median & Variance & Median & Variance & Median & Variance & & Median & Variance & Median & Variance & Median & Variance & & Median & Variance & Median & Variance & Median & Variance \\
\hline$N=200$ & & & & & & & & & & & & & & & & & & & & & \\
\hline $\mathrm{y}_{\mathrm{t}-1}$ & 0.95 & 0.95000 & 0.00586 & 0.94907 & 0.00024 & 0.95641 & 0.00136 & 0.50 & 0.51392 & 0.00056 & 0.50828 & 0.00227 & 0.53691 & 0.00200 & 0.30 & 0.31301 & 0.00058 & 0.32632 & 0.00151 & 0.33918 & 0.00299 \\
\hline $\mathrm{x}_{1}$ & 0.07 & 0.07003 & 0.00004 & 0.07037 & 0.00002 & 0.06381 & 0.00081 & 0.07 & 0.07016 & 0.00001 & 0.07107 & 0.00002 & 0.06747 & 0.00074 & 0.07 & 0.06952 & 0.00001 & 0.07038 & 0.00002 & 0.06403 & 0.00066 \\
\hline $\mathrm{x}_{2}$ & 0.00 & 0.00004 & 0.00000 & 0.00001 & 0.00000 & -0.00081 & 0.00009 & 0.00 & -0.00003 & 0.00000 & 0.00003 & 0.00001 & 0.00066 & 0.00015 & 0.00 & 0.00007 & 0.00000 & -0.00010 & 0.00000 & 0.00009 & 0.00008 \\
\hline$x_{3}$ & 0.00 & 0.00001 & 0.00000 & 0.00002 & 0.00000 & -0.00042 & 0.00016 & 0.00 & 0.00000 & 0.00000 & -0.00008 & 0.00001 & 0.00000 & 0.00009 & 0.00 & 0.00000 & 0.00000 & 0.00007 & 0.00000 & -0.00057 & 0.00015 \\
\hline $\mathrm{x}_{4}$ & -0.09 & -0.09019 & 0.00003 & -0.09031 & 0.00001 & -0.08839 & 0.00079 & -0.09 & -0.08976 & 0.00001 & -0.08976 & 0.00002 & -0.09138 & 0.00070 & -0.09 & -0.09083 & 0.00001 & -0.09052 & 0.00002 & -0.08948 & 0.00055 \\
\hline $\mathrm{x}_{5}$ & 0.00 & 0.00008 & 0.00000 & 0.00002 & 0.00001 & -0.00009 & 0.00005 & 0.00 & 0.00001 & 0.00000 & -0.00003 & 0.00000 & 0.00060 & 0.00004 & 0.00 & 0.00003 & 0.00000 & -0.00015 & 0.00001 & -0.00016 & 0.00010 \\
\hline $\mathrm{x}_{6}$ & 0.10 & 0.09953 & 0.00003 & 0.09898 & 0.00002 & 0.09978 & 0.00068 & 0.10 & 0.10044 & 0.00001 & 0.10002 & 0.00002 & 0.10080 & 0.00045 & 0.10 & 0.10020 & 0.00001 & 0.09973 & 0.00002 & 0.09942 & 0.00060 \\
\hline $\mathrm{w}_{1}$ & 0.00 & 0.00028 & 0.00001 & 0.00059 & 0.00003 & 0.00132 & 0.00040 & 0.00 & 0.00022 & 0.00001 & 0.00036 & 0.00002 & -0.00073 & 0.00024 & 0.00 & 0.00026 & 0.00001 & 0.00016 & 0.00001 & 0.00007 & 0.00032 \\
\hline $\mathrm{w}_{2}$ & -0.10 & -0.09635 & 0.00127 & -0.09712 & 0.00006 & -0.10031 & 0.00041 & -0.10 & -0.09567 & 0.00003 & -0.09692 & 0.00006 & -0.09428 & 0.00028 & -0.10 & -0.09809 & 0.00004 & -0.09405 & 0.00004 & -0.09610 & 0.00035 \\
\hline$N=500$ & & & & & & & & & & & & & & & & & & & & & \\
\hline$y_{t-1}$ & 0.95 & 0.95000 & 0.00001 & 0.95010 & 0.00001 & 0.95674 & 0.00048 & 0.50 & 0.50875 & 0.00023 & 0.50203 & 0.00065 & 0.51779 & 0.00070 & 0.30 & 0.30944 & 0.00020 & 0.31437 & 0.00044 & 0.32502 & 0.00072 \\
\hline $\mathrm{x}_{1}$ & 0.07 & 0.07012 & 0.00000 & 0.06996 & 0.00001 & 0.06880 & 0.00018 & 0.07 & 0.06966 & 0.00000 & 0.07034 & 0.00001 & 0.06715 & 0.00017 & 0.07 & 0.07011 & 0.00000 & 0.06975 & 0.00001 & 0.07337 & 0.00014 \\
\hline $\mathrm{x}_{2}$ & 0.00 & -0.00001 & 0.00000 & 0.00004 & 0.00000 & -0.00031 & 0.00004 & 0.00 & -0.00001 & 0.00000 & 0.00002 & 0.00000 & -0.00017 & 0.00002 & 0.00 & 0.00003 & 0.00000 & -0.00007 & 0.00000 & -0.00015 & 0.00003 \\
\hline$x_{3}$ & 0.00 & -0.00004 & 0.00000 & 0.00009 & 0.00000 & -0.00018 & 0.00002 & 0.00 & 0.00002 & 0.00000 & -0.00008 & 0.00000 & -0.00009 & 0.00003 & 0.00 & 0.00001 & . 00000 & -0.00003 & 0.00000 & -0.00060 & 0.00003 \\
\hline $\mathrm{x}_{4}$ & -0.09 & -0.09016 & 0.00000 & -0.09064 & 0.00001 & -0.09000 & 0.00014 & -0.09 & -0.09021 & 0.00000 & -0.09006 & 0.00001 & -0.09240 & 0.00017 & -0.09 & -0.09063 & 0.00000 & -0.09086 & 0.00001 & -0.08872 & 0.00017 \\
\hline $\mathrm{x}_{5}$ & 0.00 & 0.00003 & 0.00000 & 0.00001 & 0.00000 & 0.00015 & 0.00007 & 0.00 & -0.00004 & 0.00000 & 0.00008 & 0.00000 & 0.00003 & 0.00002 & 0.00 & 0.00002 & 0.00000 & -0.00005 & 0.00000 & -0.00013 & 0.00002 \\
\hline $\mathrm{x}_{6}$ & 0.10 & 0.10020 & 0.00000 & 0.09925 & 0.00001 & 0.10256 & 0.00016 & 0.10 & 0.10023 & 0.00000 & 0.09945 & 0.00001 & 0.10119 & 0.00015 & 0.10 & 0.09986 & 0.00000 & 0.09960 & 0.00001 & 0.09951 & 0.00016 \\
\hline $\mathrm{w}_{1}$ & 0.00 & 0.00005 & 0.00000 & 0.00019 & 0.00000 & 0.00058 & 0.00004 & 0.00 & 0.00008 & 0.00000 & 0.00008 & 0.00000 & -0.00012 & 0.00001 & 0.00 & 0.00017 & 0.00000 & 0.00004 & 0.00000 & 0.00007 & 0.00003 \\
\hline $\mathrm{w}_{2}$ & -0.10 & -0.09861 & 0.00001 & -0.09897 & 0.00002 & -0.10023 & 0.00004 & -0.10 & -0.09774 & 0.00001 & -0.09811 & 0.00002 & -0.09741 & 0.00002 & -0.10 & -0.09876 & 0.00002 & -0.09795 & 0.00002 & -0.09770 & 0.00003 \\
\hline$N=10$ & & & & & & & & & & & & & & & & & & & & & \\
\hline $\mathrm{y}_{\mathrm{t}-1}$ & 0.95 & 0.95008 & 0.00000 & 0.94962 & 0.00001 & 0.95328 & 00024 & 0.50 & 0.50535 & 0.00012 & 0.50579 & 0.00023 & 1277 & 0.00026 & 0.30 & 0.30580 & 00010 & 0.30667 & 0.00022 & 0.31089 & 0.00027 \\
\hline $\mathrm{x}_{1}$ & 0.07 & 0.07005 & 0.00000 & 0.07035 & 0.00000 & 0.06885 & 0.00006 & 0.07 & 0.07000 & 0.00000 & 0.07026 & 0.00000 & 0.07080 & 0.00009 & 0.07 & 0.06988 & 0.00000 & 0.06904 & 0.00000 & 0.07201 & 0.00009 \\
\hline $\mathrm{x}_{2}$ & 0.00 & 0.00002 & 0.00000 & 0.00002 & 0.00000 & -0.00019 & 0.00001 & 0.00 & 0.00002 & 0.00000 & 0.00004 & 0.00000 & -0.00002 & 0.00001 & 0.00 & 0.00002 & 0.00000 & -0.00001 & 0.00000 & -0.00015 & 0.00002 \\
\hline$x_{3}$ & 0.00 & -0.00002 & 0.00000 & 0.00007 & 0.00000 & -0.00021 & 0.00001 & 0.00 & 0.00002 & 0.00000 & -0.00009 & 0.00000 & -0.00002 & 0.00002 & 0.00 & 0.00000 & 0.00000 & 0.00004 & 0.00000 & -0.00035 & 0.00001 \\
\hline $\mathrm{x}_{4}$ & -0.09 & -0.09038 & 0.00000 & -0.09047 & 0.00000 & -0.08988 & 0.00007 & -0.09 & -0.08995 & 0.00000 & -0.08961 & 0.00000 & -0.09250 & 0.00006 & -0.09 & -0.09011 & 00000 & -0.09005 & 0.00000 & -0.08765 & 0.00008 \\
\hline $\mathrm{x}_{5}$ & 0.00 & 0.00004 & 0.00000 & 0.00000 & 0.00000 & -0.00007 & 0.00002 & 0.00 & -0.00003 & 0.00000 & 0.00000 & 0.00000 & -0.00016 & 0.00001 & 0.00 & 0.00002 & 000 & -0.00002 & 0000 & -0.00011 & 0.00001 \\
\hline $\mathrm{x}_{6}$ & 0 & 008 & 00 & 9951 & 000 & 66 & 007 & & 034 & 000 & 0.10010 & 00 & 064 & & 0.10 & 022 & 000 & 9948 & 000 & 0.09632 & 0.00008 \\
\hline $\mathrm{w}_{1}$ & 0.00 & 0.00004 & 0.00000 & 0.00021 & 0.00000 & 0.00058 & 0.00002 & 0.00 & 0.00001 & 0.00000 & -0.00002 & 0.00000 & -0.00019 & 0.00000 & 0.00 & 0.00001 & 0.00000 & -0.00002 & 0.00000 & 0.00000 & 0.00001 \\
\hline $\mathrm{w}_{2}$ & -0.10 & -0.09959 & 0.00001 & -0.09913 & 0.00001 & -0.10109 & 0.00002 & -0.10 & -0.09808 & 0.00001 & -0.09848 & 0.00001 & -0.09808 & 0.00001 & -0.10 & -0.09876 & 0.00001 & -0.09915 & 0.00001 & -0.09837 & 0.00001 \\
\hline$N=20$ & & & & & & & & & & & & & & & & & & & & & \\
\hline $\mathrm{y}_{\mathrm{t}-1}$ & 0.95 & 0.94994 & 0.00000 & 0.94972 & 0.00000 & 0.95469 & 0.00011 & 0.50 & 0.50489 & 0.00004 & 0.49944 & 0.00011 & 0.51104 & 0.00011 & 0.30 & 0.30273 & 0.00006 & 0.30012 & 0.00010 & 0.31344 & 0.00015 \\
\hline $\mathrm{x}_{1}$ & 97 & 0.07026 & 0.00000 & 0.07036 & 0.00000 & 0.06932 & 0.00004 & 0.07 & 0.06967 & 0.00000 & 0.07018 & 0.00000 & 0.07050 & & 0.07 & 0.06978 & & 0.06914 & 0.00000 & 0.07292 & 0.00004 \\
\hline $\mathrm{x}_{2}$ & 0.00 & 0.00000 & 0.00000 & 0.00000 & 0.00000 & -0.00037 & 0.00001 & 0.00 & 0.00000 & 0.00000 & 0.00002 & 000 & -0.00002 & & 0.00 & 0.00001 & 0.00000 & -0.00002 & 0.00000 & -0.00004 & 0.00000 \\
\hline$x_{3}$ & 0.00 & -0.00003 & 0.00000 & 0.00006 & 000 & -0.00013 & 000 & 0.00 & 0.00002 & 000 & -0.00005 & 000 & -0.00004 & 0.00000 & 0.00 & -0.00001 & 000 & 0.00003 & 0.00000 & -0.00031 & 0.00001 \\
\hline $\mathrm{x}_{4}$ & -0.09 & -0.09020 & 0.00000 & -0.09000 & 0.00000 & -0.09002 & 0.00003 & -0.09 & -0.09004 & 0.00000 & -0.08991 & 0.00000 & -0.09076 & 0.00003 & -0.09 & -0.09018 & 0.00000 & -0.09040 & 0.00000 & -0.08852 & 0.00004 \\
\hline $\mathrm{x}_{5}$ & 0.00 & 0.00005 & 0.00000 & 0.00000 & 0.00000 & -0.00006 & 0.00000 & 0.00 & -0.00002 & 0.00000 & 0.00001 & 0.00000 & -0.00005 & 0.00000 & 0.00 & 0.00002 & 0.00000 & -0.00002 & 0.00000 & -0.00007 & 0.00000 \\
\hline $\mathrm{x}_{6}$ & 0.10 & 0.10012 & 0.00000 & 0.09952 & 0.00000 & 0.10034 & 004 & 0.10 & 0.10052 & 000 & 0.09990 & 000 & 0.09883 & & 0.10 & 0.10024 & 000 & 0.09974 & 0.00000 & 0.09679 & 0.00003 \\
\hline $\mathrm{w}_{1}$ & 0.00 & 0001 & & 013 & & & & 0.00 & -0.00002 & & -0.00001 & & -0.00006 & & 0.00 & 0.00003 & & -0.00002 & 000 & -0.00003 & 0.00000 \\
\hline$w_{2}$ & -0.10 & -0.09988 & 0.00000 & -0.10014 & 0.00000 & -0.10043 & 0.00001 & -0.10 & -0.09849 & 0.00000 & -0.09920 & 0.00000 & -0.09881 & 0.00000 & -0.10 & -0.09941 & 0.00000 & -0.09969 & 0.00000 & -0.09884 & 0.00000 \\
\hline
\end{tabular}

1. The error terms are constructed using discrete distributions (see section IV.A.). 
Figure 1: Posterior Densities for the Probabilities in Table 1
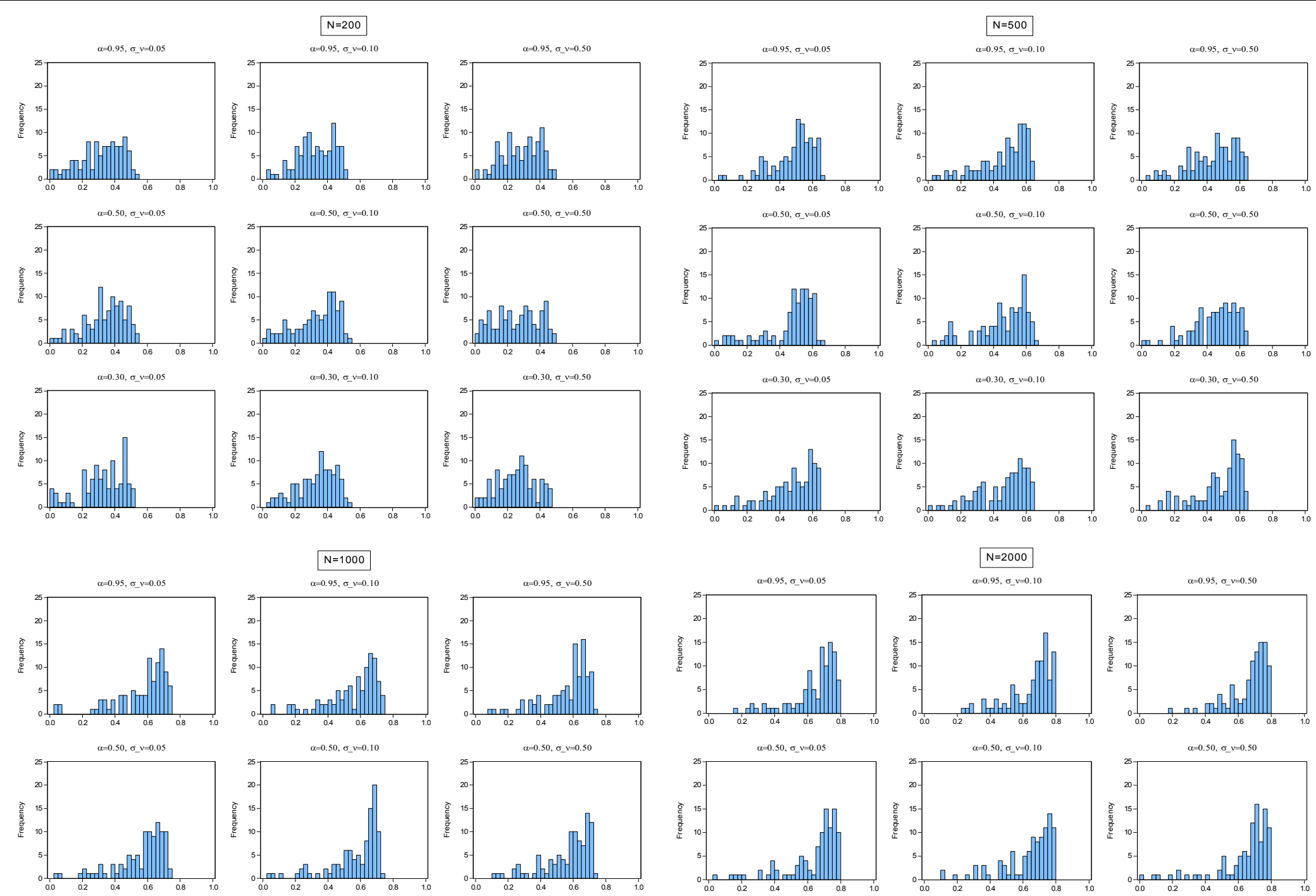

$\alpha=0.50, \sigma_{-}=0.50$
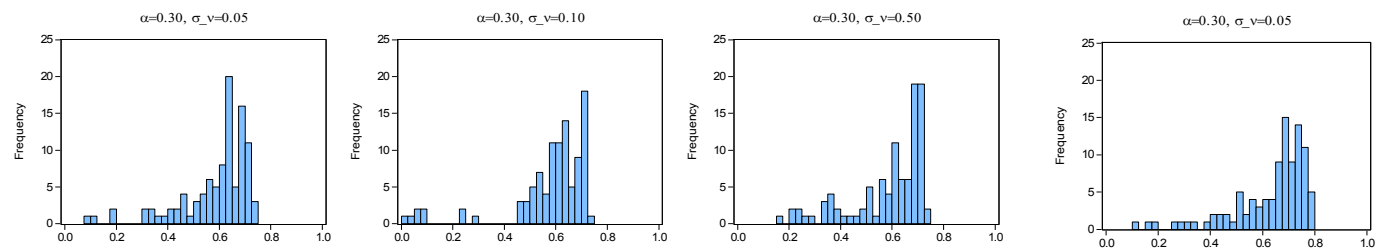

$\alpha=0.30, \sigma_{-}=0.1$
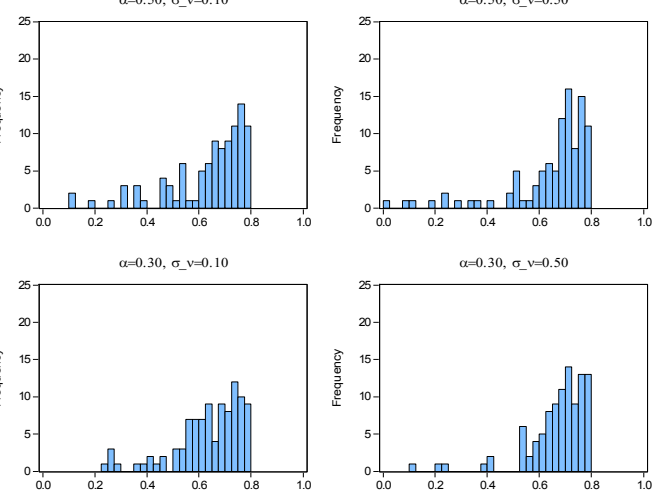

1. For the idiosyncratic error term, $\eta_{i} \sim \mathrm{N}\left(0, \sigma_{\eta}{ }^{2}\right)$ where $\sigma_{\eta}=0.10$. 2. The error term is normally distributed $v_{t} \sim \mathrm{N}\left(0, \sigma_{v}{ }^{2}\right)$. 
Figure 2: Posterior Densities for the Probabilities in Table 2
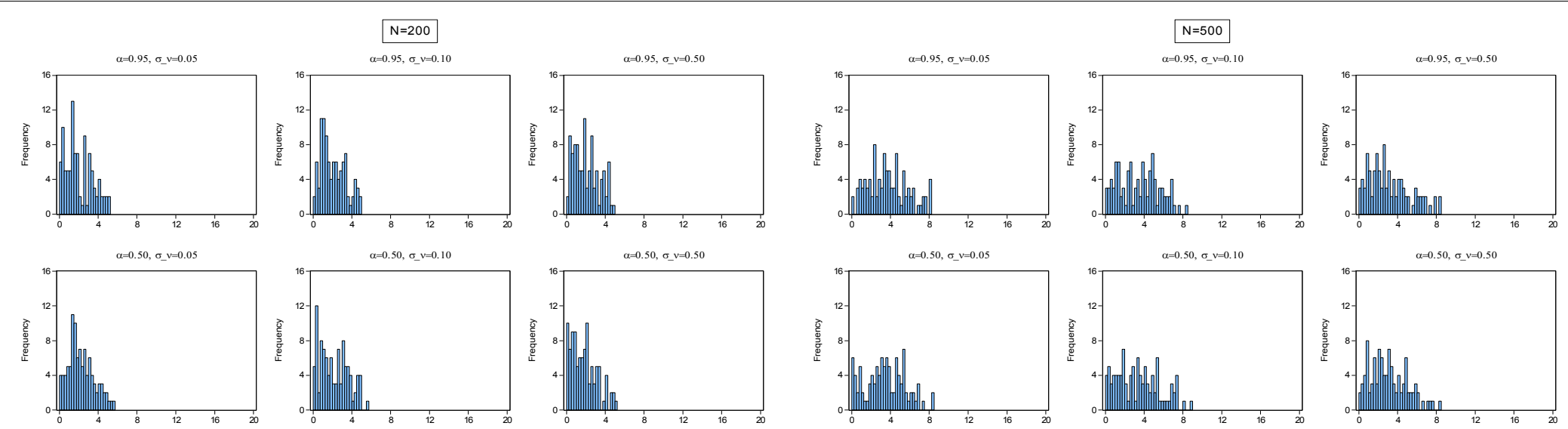

$\alpha=0.50, \sigma v=0.50$
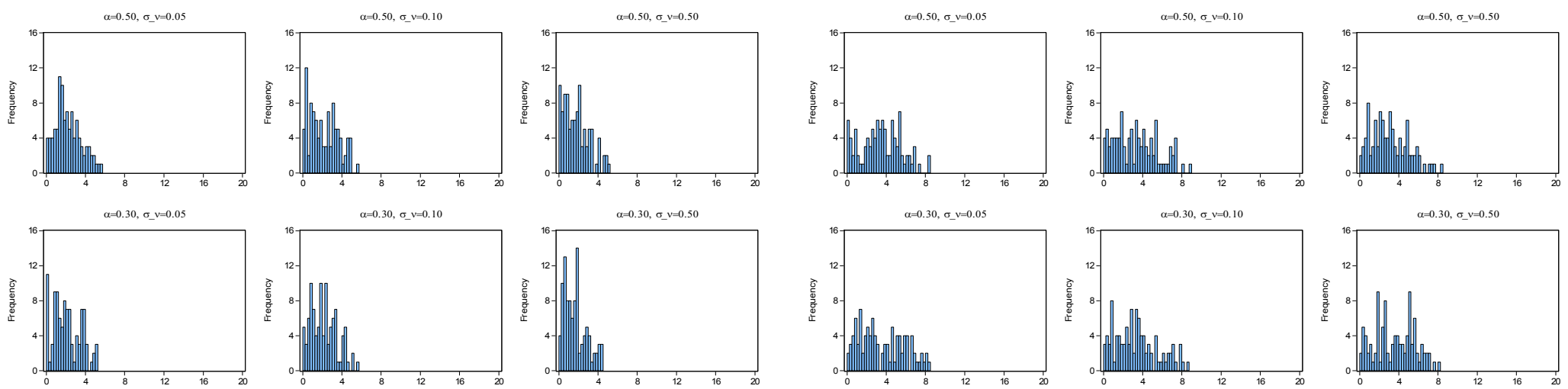

$\alpha=0.30, \sigma_{-}=0.50$
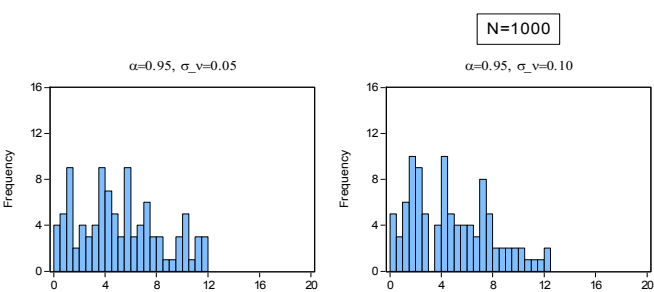

$\alpha=0.95, \sigma_{-}=0.50$
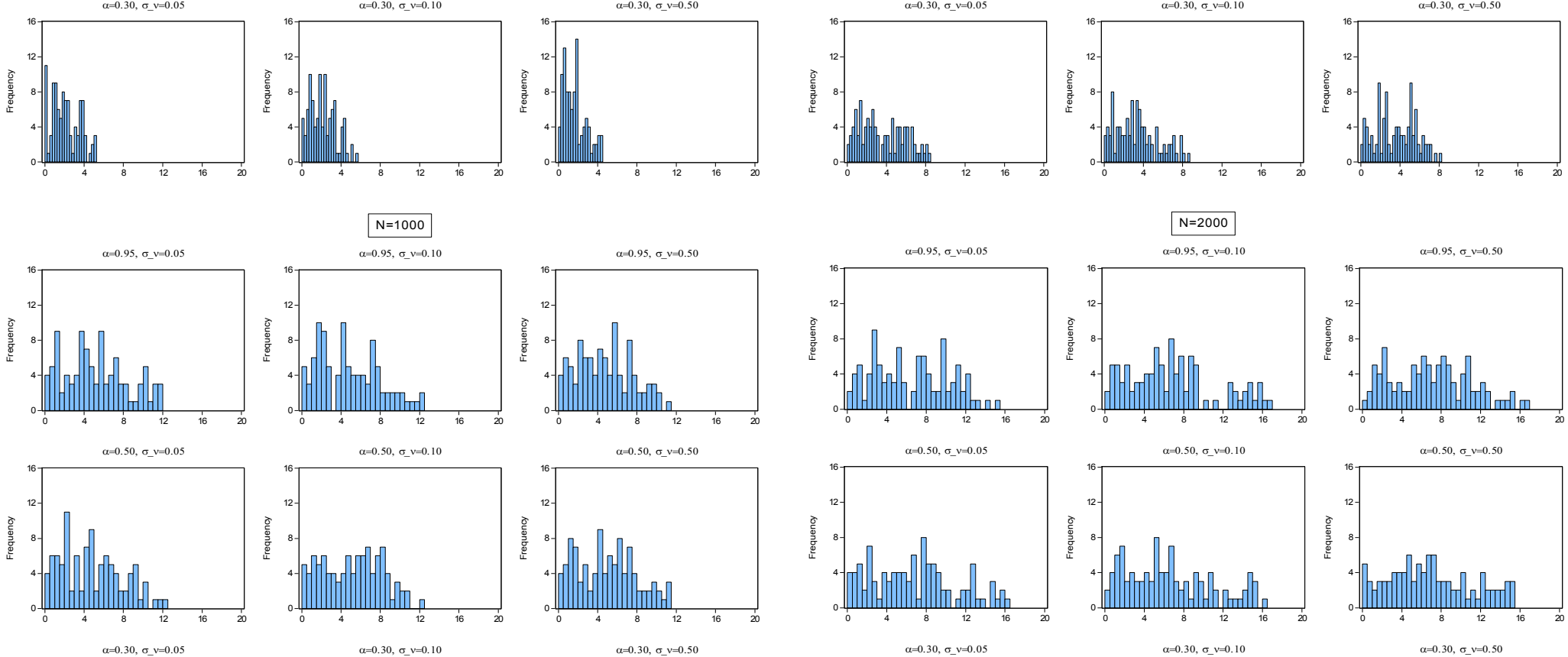

$\alpha=0.30, \sigma_{-}=0.05$

$\alpha=0.30, \sigma \_v=0.10$
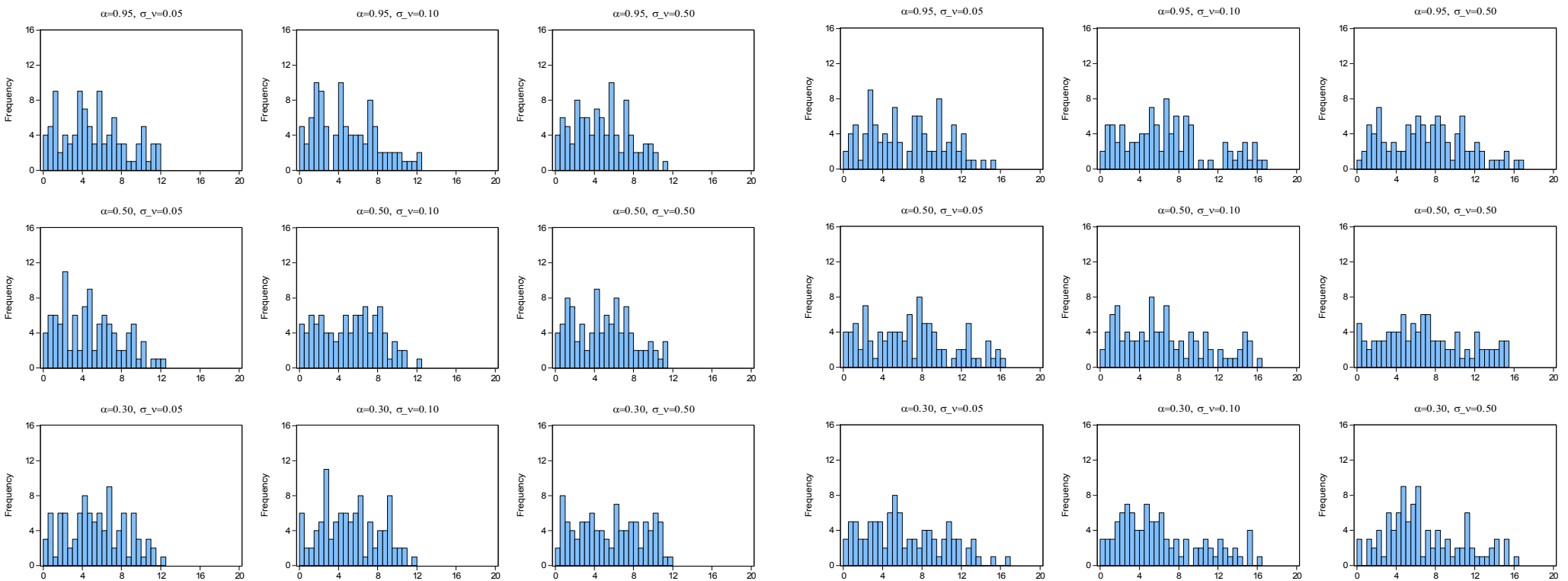

$\alpha=0.30, \sigma_{-} \vee=0.50$

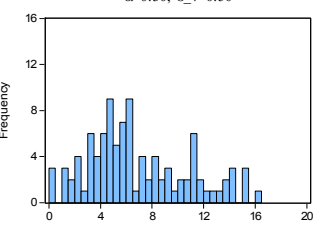

1. For the idiosyncratic error term, $\eta_{i} \sim \mathrm{N}\left(0, \sigma_{\eta}{ }^{2}\right)$ where $\sigma_{\eta}=0.10$.

2. The error term is normally distributed $\mathrm{v}_{t} \sim \mathrm{N}\left(0, \sigma_{v}{ }^{2}\right)$. 
Figure 3: Box Plots for Parameters in Table 5

BMA coefficients' estimated values for $\alpha=0.95, \sigma_{v}=0.1$ and various $N$

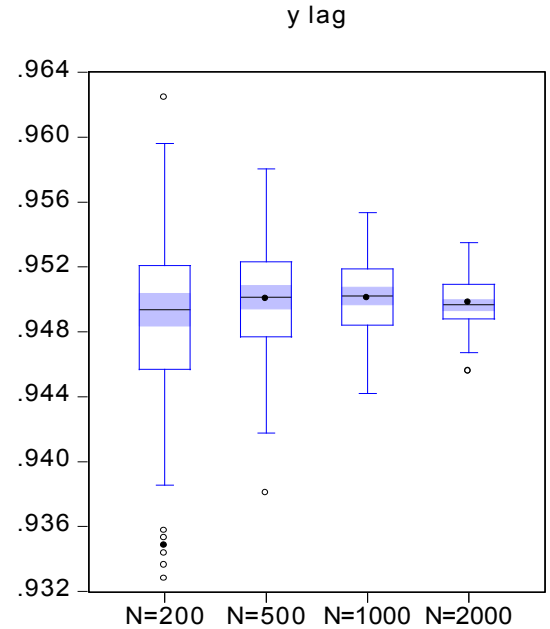

$\mathrm{x} 3$

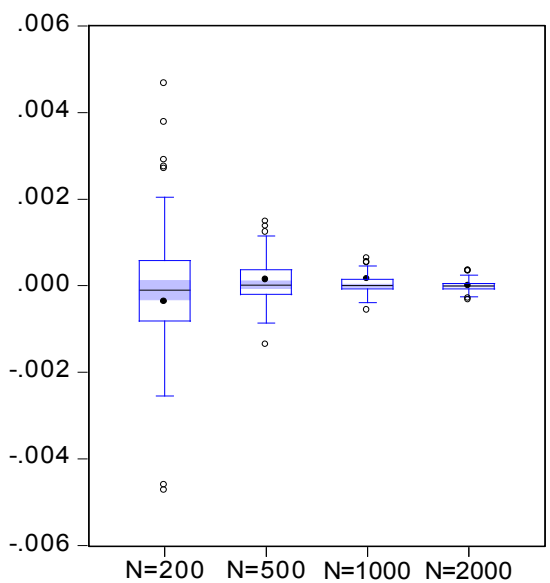

$x 6$

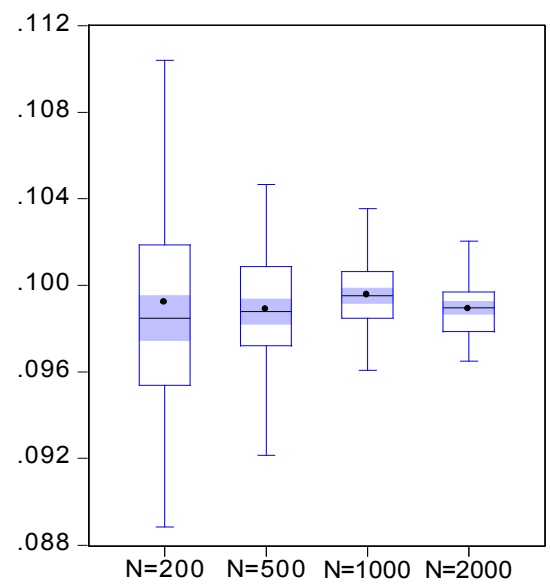

$x 1$

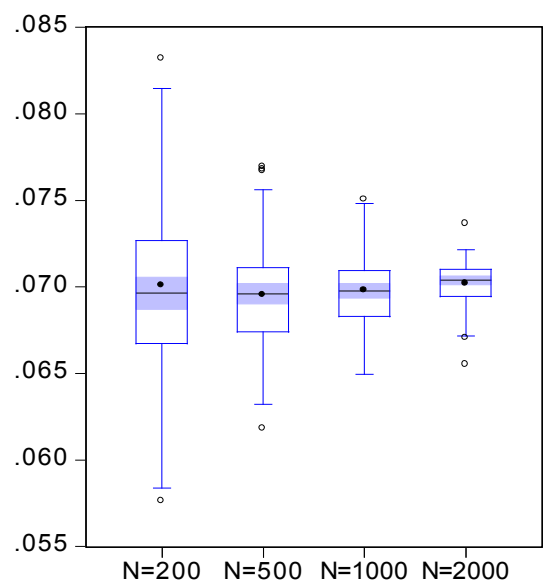

$\mathrm{x} 4$

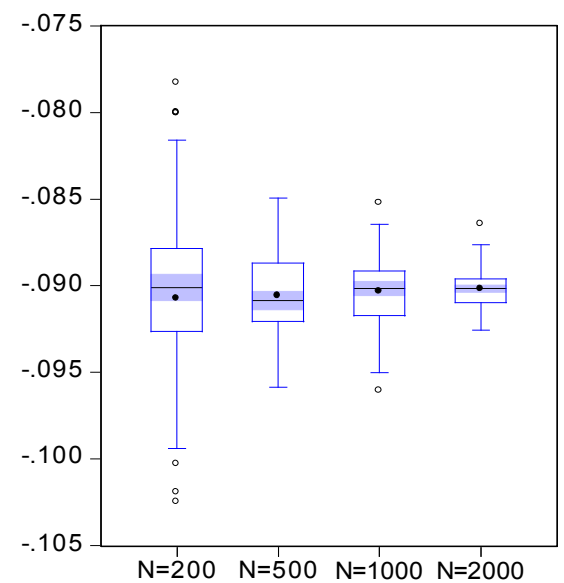

$N=200 \quad N=500 \quad N=1000 \quad N=2000$ x2

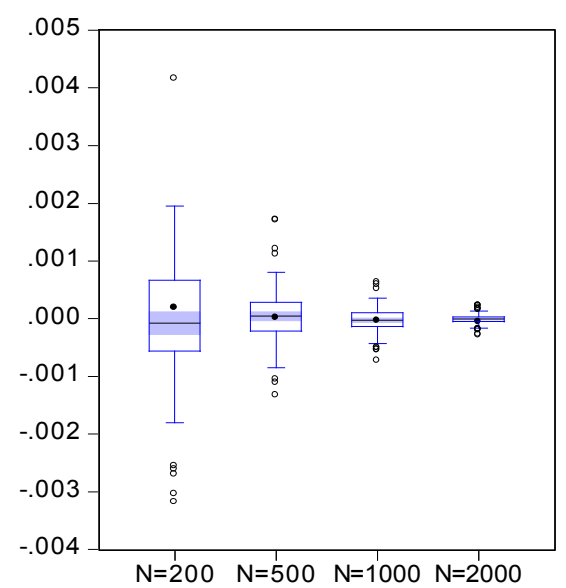

x5

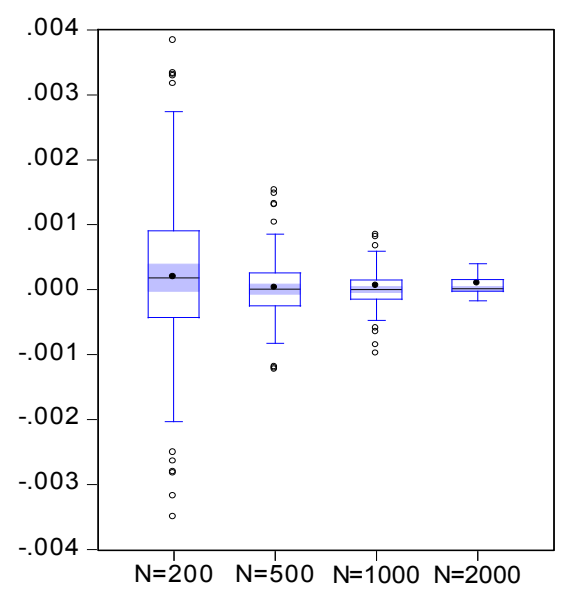

w2

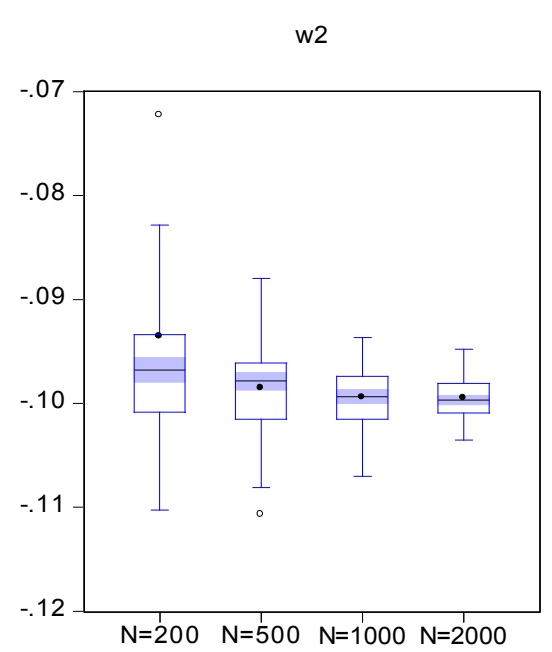

1. For the idiosyncratic error term, $\eta_{i} \sim \mathrm{N}\left(0, \sigma_{\eta}{ }^{2}\right)$ where $\sigma_{\eta}=0.10$.

2. The error term is normally distributed $v_{i t} \sim \mathrm{N}\left(0, \sigma_{v}{ }^{2}\right)$.

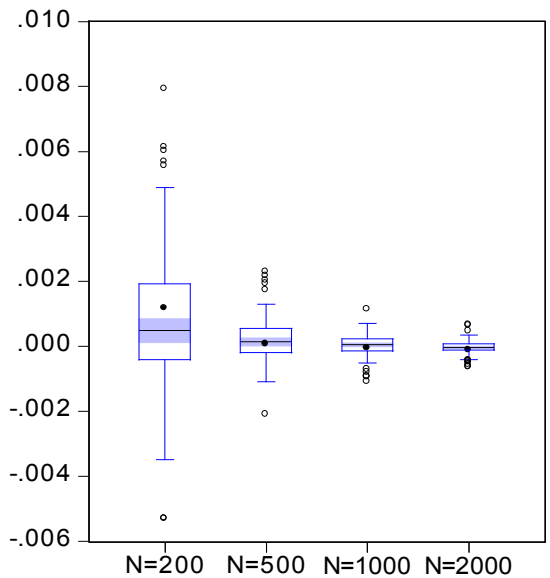


Figure 3: Posterior Densities for the Probabilities in Table 6
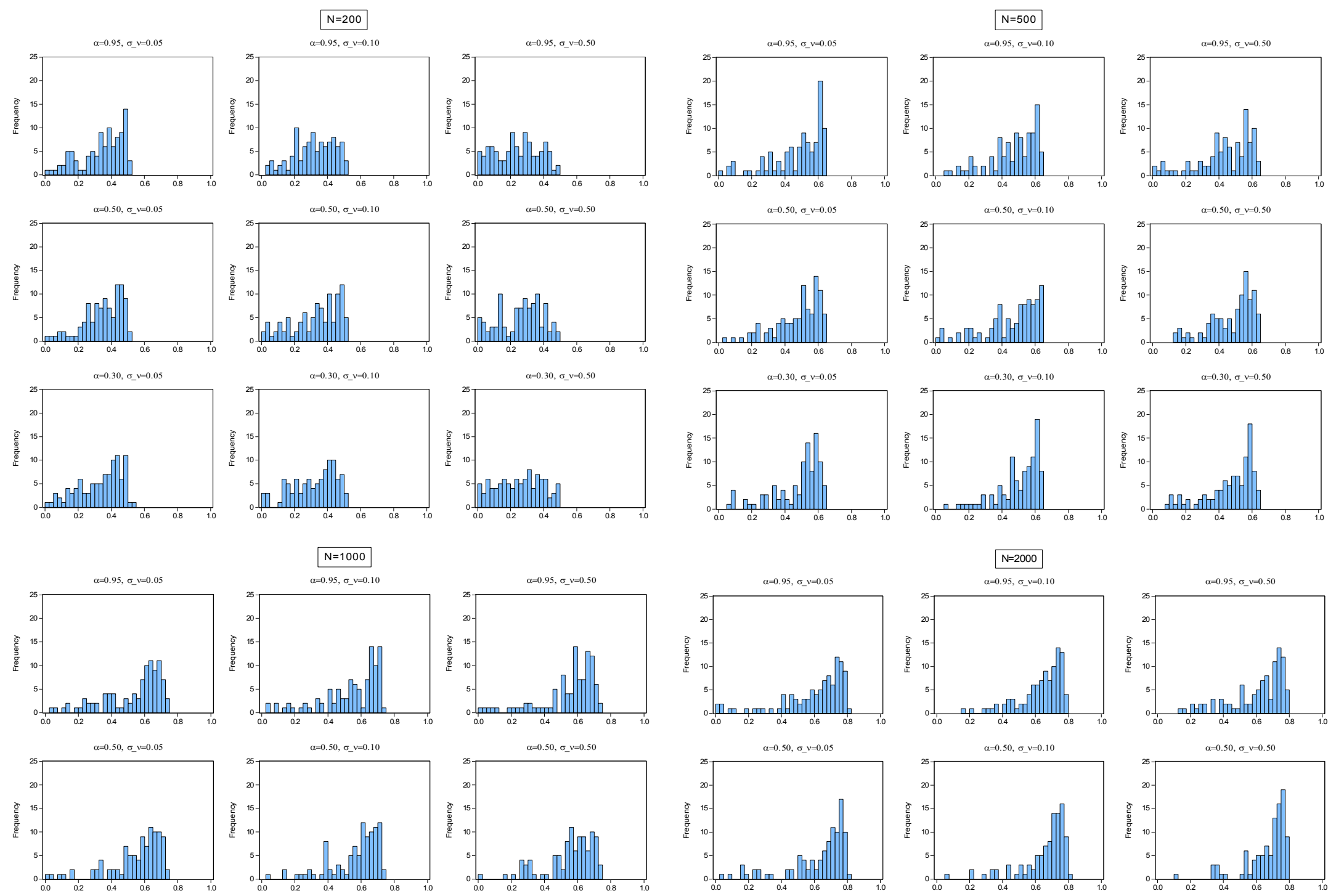

$\alpha=0.50, \sigma_{-} \vee=0.10$

$\alpha=0.50, \sigma \vee=0.50$

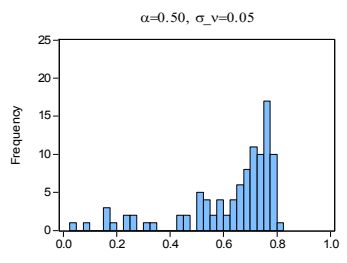

$\alpha=0.50, \sigma_{-} \vee=0.10$

$\alpha=0.50, \sigma_{-} \vee=0.50$
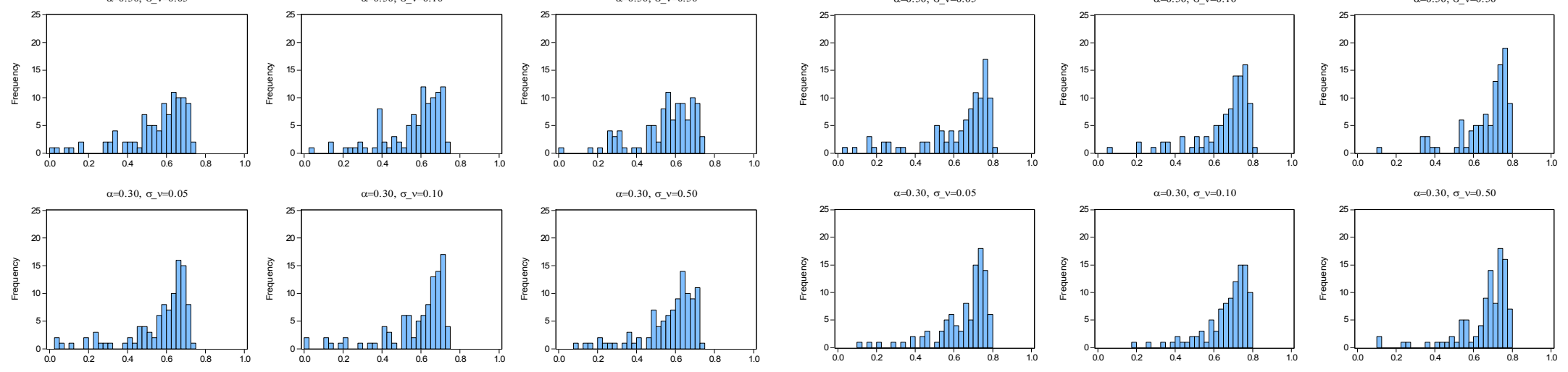
Figure 4: Posterior Densities for the Probabilities in Table 7
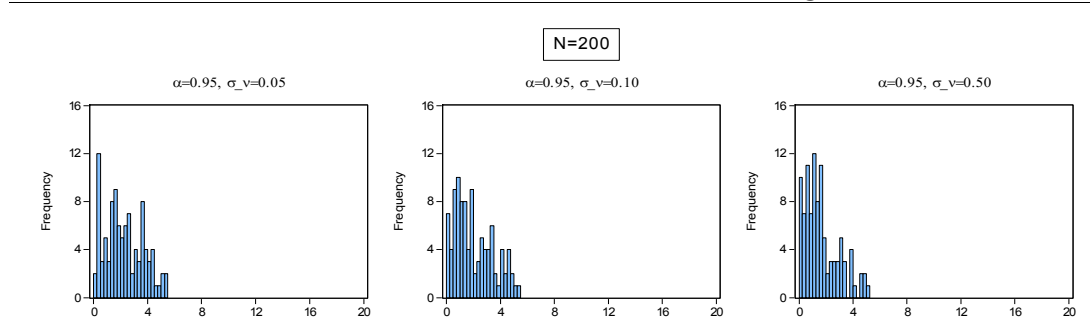

$\alpha=0.50, \sigma_{-} \vee=0.05$

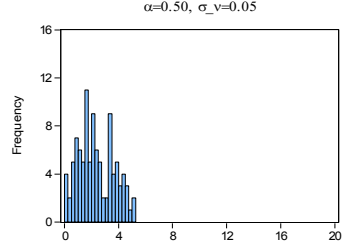

$\alpha=0.30, \sigma_{-}=0.05$

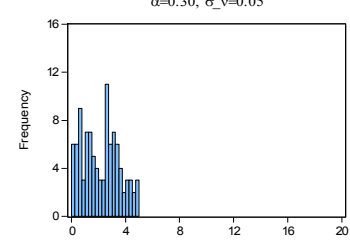

$\alpha=0.95, \sigma \_v=0.05$

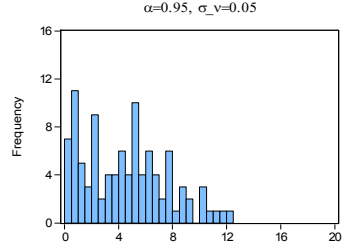

$\alpha=0.50, \sigma \_\vee=0.0$

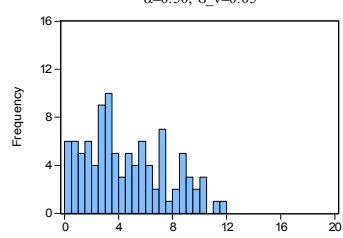

$\alpha=0.30, \sigma=0=0.05$

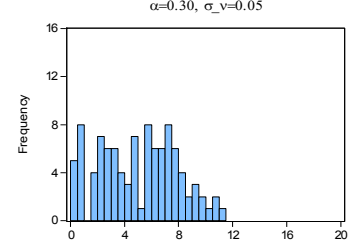

$\alpha=0.50, \sigma_{-} v=0.50$

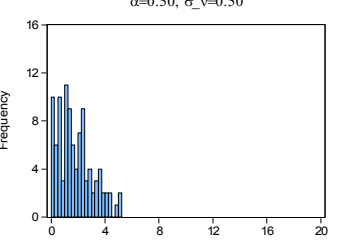

$\alpha=0.30, \sigma_{-} v=0.50$

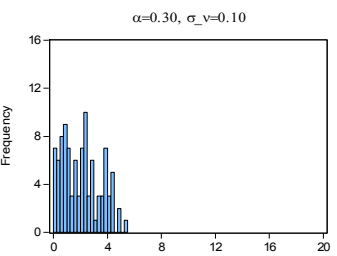

$\mathrm{N}=1000$

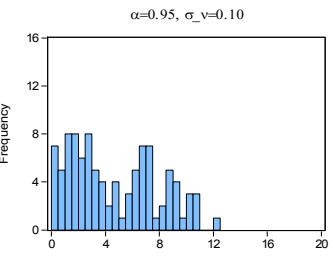

$\alpha=0.50, \sigma_{-}=0.1$

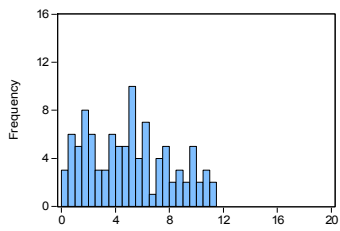

$\alpha=0.30, \sigma_{-}=0.10$

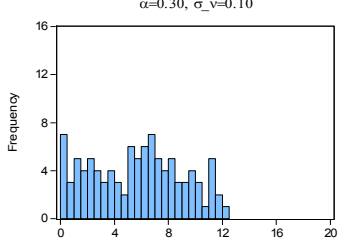

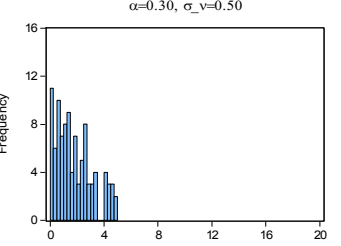

$\alpha=0.95, \sigma_{-}=0.50$

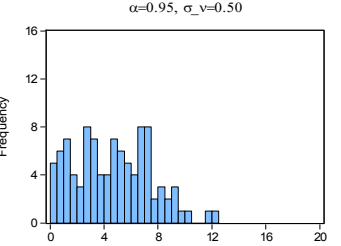

$\alpha=0.50, \sigma_{-}=0.50$

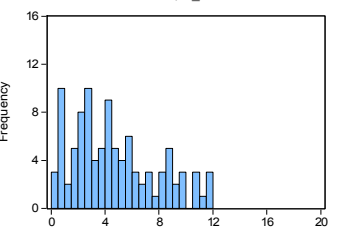

$\alpha=0.30, \sigma_{-}=0.50$

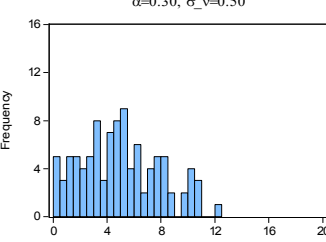

$\mathrm{N}=500$
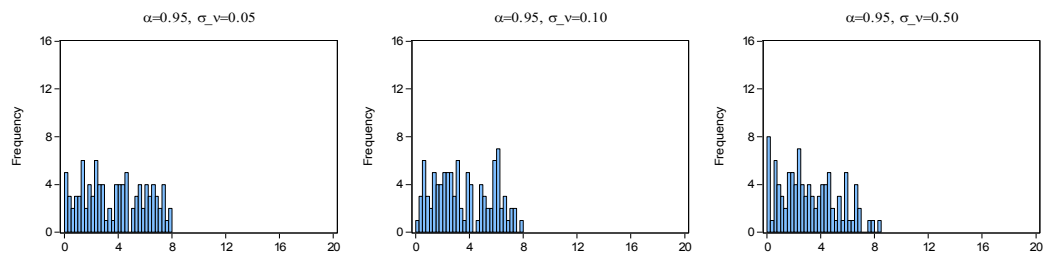

$\alpha=0.50, \sigma \_v=0.05$

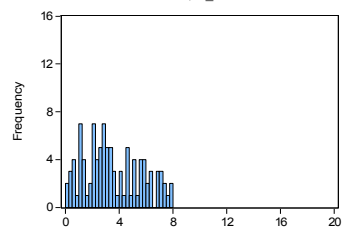

$u=0.50, \sigma_{-} \vee=0.10$
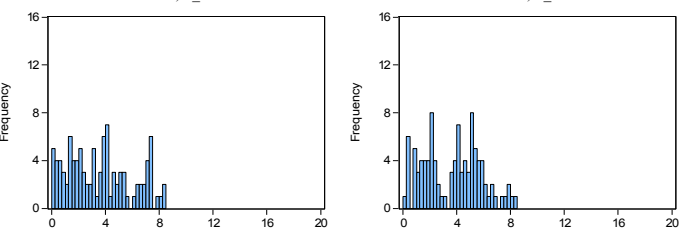

$\alpha=0.30, \sigma_{-} v=0.10$
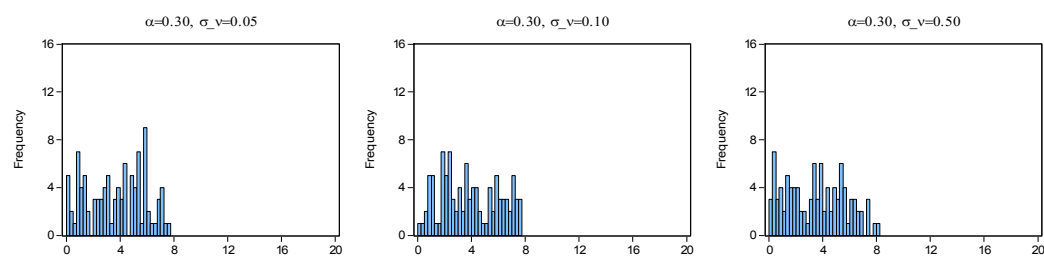

$\mathrm{N}=2000$
$\alpha=0.95, \sigma v=0$.
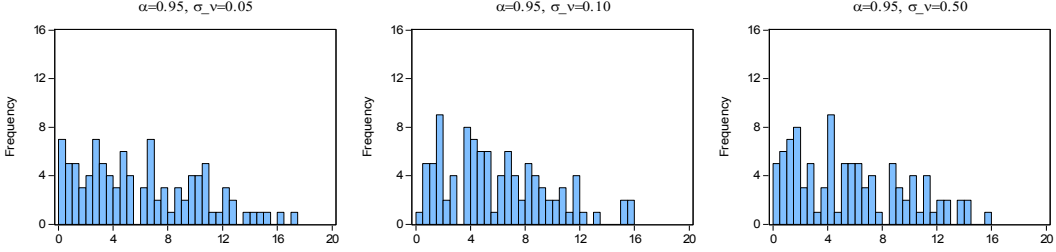

$\alpha=0.50, \sigma_{-}=0.05$

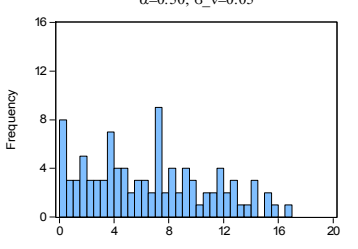

$\alpha=0.50, \sigma_{-} v=0.1$

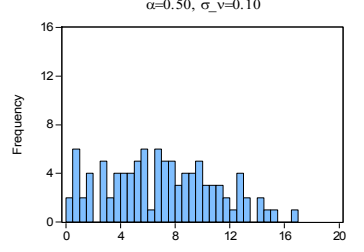

$=0.50, \sigma_{-}=0.5$

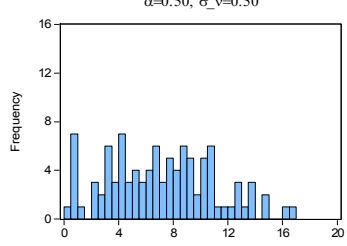

$\alpha=0.30, \sigma_{-} v=0.05$

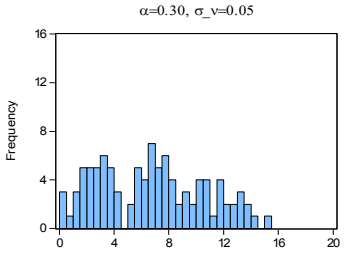

$\alpha=0.30, \sigma \vee v=0.1$

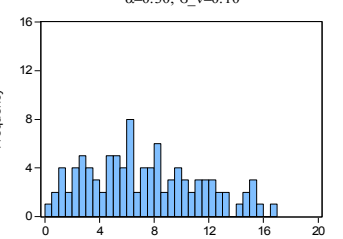

$=0.30, \sigma_{-}=0.5$

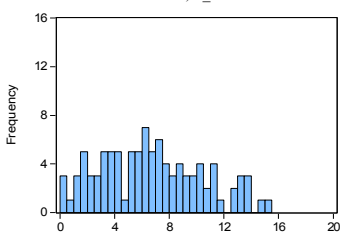


Figure 6: Box Plots for Parameters in Table 10

BMA coefficients' estimated values for $\alpha=0.95, \sigma_{v}=0.1$ and various $N$
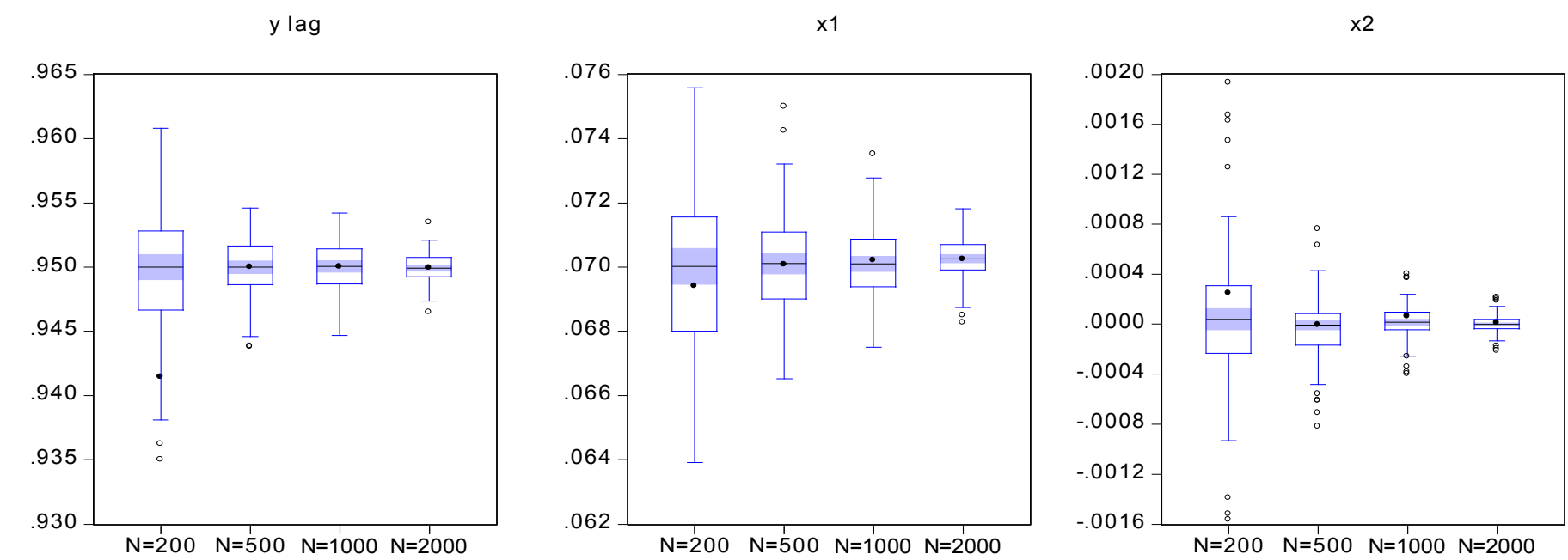

$\times 3$
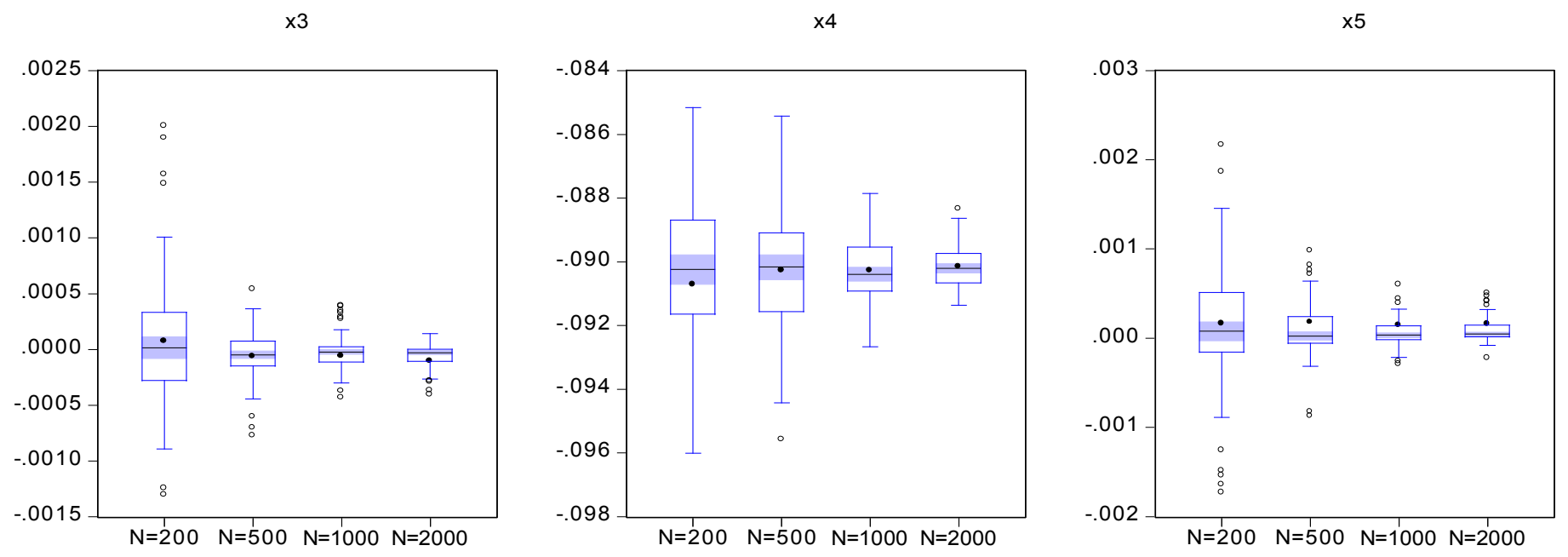

x6

w1
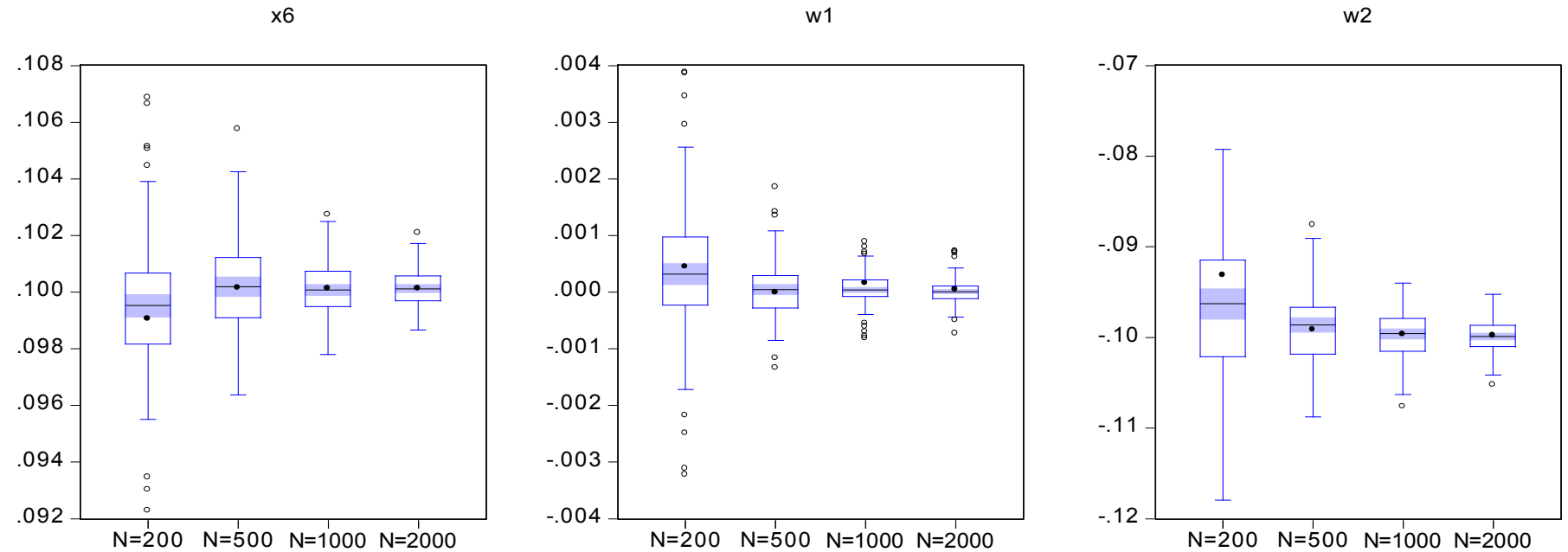